\title{
Effects of network resolution on topological properties of human neocortex
}

Romero-Garcia, Rafael; Atienza, Mercedes; Clemmensen, Line Katrine Harder; Cantero, Jose L.

Published in:

Neurolmage

Link to article, DOI:

10.1016/j.neuroimage.2011.10.086

Publication date:

2012

Link back to DTU Orbit

Citation (APA):

Romero-Garcia, R., Atienza, M., Clemmensen, L. K. H., \& Cantero, J. L. (2012). Effects of network resolution on topological properties of human neocortex. Neurolmage, 59(4), 3522-3532.

https://doi.org/10.1016/j.neuroimage.2011.10.086

\section{General rights}

Copyright and moral rights for the publications made accessible in the public portal are retained by the authors and/or other copyright owners and it is a condition of accessing publications that users recognise and abide by the legal requirements associated with these rights.

- Users may download and print one copy of any publication from the public portal for the purpose of private study or research.

- You may not further distribute the material or use it for any profit-making activity or commercial gain

- You may freely distribute the URL identifying the publication in the public portal

If you believe that this document breaches copyright please contact us providing details, and we will remove access to the work immediately and investigate your claim 


\title{
Effects of network resolution on topological properties of human neocortex
}

\author{
Rafael Romero-Garcia ${ }^{1}$, Mercedes Atienza ${ }^{1}$, Line H. Clemmensen ${ }^{2}$, Jose L. Cantero ${ }^{1 *}$ \\ ${ }^{1}$ Laboratory of Functional Neuroscience, Spanish Network of Excellence for Research \\ on Neurodegenerative Diseases (CIBERNED), University Pablo de Olavide, Seville, \\ Spain \\ ${ }^{2}$ Technical University of Denmark, Informatics and Mathematical Modelling, DK-2800 \\ Lyngby, Denmark
}

\section{Corresponding author:}

Jose L. Cantero, Ph.D.

Laboratory of Functional Neuroscience

University Pablo de Olavide

Carretera de Utrera Km 1,

41013 - Seville (Spain)

Phone: +34 954977433

Fax: +34 954349151

E-mail: jlcanlor@upo.es 


\begin{abstract}
Graph theoretical analyses applied to neuroimaging datasets have provided valuable insights into the large-scale anatomical organization of the human neocortex. Most of these studies were performed with different cortical scales leading to cortical networks with different levels of small-world organization. The present study investigates how resolution of thickness-based cortical scales impacts on topological properties of human anatomical cortical networks. To this end, we designed a novel approach aimed at determining the best trade-off between small-world attributes of anatomical cortical networks and the number of cortical regions included in the scale. Results revealed that schemes comprising 540-599 regions (surface areas spanning between 250-275 $\mathrm{mm}^{2}$ ) at sparsities below 10\% showed a superior balance between small-world organization and the size of the cortical scale employed. Furthermore, we found that the cortical scale representing the best trade-off (599 regions) was more resilient to targeted attacks than atlas-based schemes (Desikan-Killiany atlas, 66 regions) and, most importantly, it did not differ that much from the finest cortical scale tested in the present study (1494 regions). In summary, our study confirms that topological organization of anatomical cortical networks varies with both sparsity and resolution of cortical scale, and it further provides a novel methodological framework aimed at identifying cortical schemes that maximize small-worldness with the lowest scale resolution possible.
\end{abstract}

Keywords: Cortical networks, structural connectivity, cortical scale, small-world properties; cortical thickness. 


\section{Introduction}

The repertoire of complex behaviors increases linearly with the number and size of cortical fields (Krubitzer, 2007). Enrichment of cortical fields has shown to be critical for the emergence of highly efficient anatomical networks in the mammal neocortex (Douglas and Martin, 2004). Understanding how specific cortical systems contribute to the organization of the entire neocortex will drastically improve our knowledge on anatomical basis of higher cognitive functions, and will allow us to forecast to what extent local cortical dysfunctions might affect global organization of the cortical mantle.

Graph theoretical analyses applied to neuroimaging datasets have provided novel insights into the topological properties of anatomical brain networks in health (Fan et al., 2010; Gong et al., 2009; He et al., 2007) and disease (Shu et al., 2009; Bassett et al., 2008; He et al., 2008). Schemes derived from small-world architecture have demonstrated to grasp the essence of this biological organization (Fair et al., 2009; Hagmann et al., 2008). Small-world topology is characterized by a high density of local connections together with a scarce number of links between distant regions, leading to highly efficient networks with a relatively low wiring cost and optimal adaptability to a broad range of circumstances (Travers and Milgram, 1969).

Recent evidence suggests that topological organization of anatomical brain networks are critically affected by a priori atlases (Sanabria-Diaz et al., 2010) or spatial scales derived from random nodal parcellation (Zalesky et al., 2010), raising the question of whether different scale resolution leads to different levels of small-world properties in different experimental scenarios. To approach this issue, we systematically assessed here how network resolution influences topological organization of anatomical cortical 
networks by using interregional correlations in cortical thickness as a measure of structural connectivity.

Anatomical cortical networks have been inferred from different descriptors (volume or thickness), and they often rely on pair-wise correlations between regions across individuals (Fan et al., 2010; He et al., 2009). This approach, although largely employed in small-world studies (e.g., Tian et al., 2010; Wang et al., 2009), inflates correlation values from surrounding cortical areas, which, in turn, contribute to shape artifactual network topologies. Furthermore, statistical power of network analysis is critically influenced by experimental designs integrated by a number of subjects that is smaller than the number of dependent variables, the so-called "small $n$, large $p$ " problem (Mantegna and Stanley, 2000). Covariance matrices derived from "small $n$, large $p$ " experimental designs are inaccurate and lead to over-fitted statistical models. This scenario is common when highly grained scales are being tested with small samples of subjects. To overcome this drawback, we applied here for the first time a novel shrinkage approach that not only increases the precision rate when controlling for false positives, but it also drastically reduces the computation time (Schäfer and Strimmer, 2005), which is a critical issue when using fine cerebral scales. Results of the present study will be discussed within the framework of anatomo-functional organization of the neocortical mantle underlying different cortical scales.

\section{Materials and Methods}

\section{1 Subjects}

Thirty cognitively intact elderly volunteers (17 females, mean age: $66.4 \pm 5.1 \mathrm{yr}$; Mini Mental State Examination: $28.4 \pm 0.2$ ) were recruited from the local community. They 
provided written informed consent before participating in the study. The Ethical Committee for Human Research of the University Pablo de Olavide previously approved research protocols, and experiments were conducted according to the principles expressed in the Declaration of Helsinki.

Inclusion criteria were (i) no subjective or objective memory complaints corroborated by neuropsychological exploration, (ii) CDR (Global Clinical Dementia Rating) global score of 0 (no dementia), and (iii) normal independent function judged clinically and by means of a standardized scale for the activities of daily living. None of them reported a history of neurological, psychiatric disorders and/or major medical illness.

\subsection{MRI acquisition and pre-processing}

Two high-resolution three-dimensional (3D) T1-weighted magnetization-prepared rapid gradient echo (MP-RAGE) images were acquired in the same session on a whole-body Philips Intera 1.5T MRI scanner (Philips, The Netherlands). MP-RAGE parameters were empirically optimized for gray/white contrast (repetition time $=8.5 \mathrm{~ms}$, echo time $=4 \mathrm{~ms}$, flip angle $=8^{\circ}$, matrix dimensions $256 \times 192,184$ contiguous sagittal $1.2 \mathrm{~mm}$ thick slices, time per acquisition $=5.4 \mathrm{~min}$ ).

\subsection{Cortical surface reconstruction and cortical thickness estimation}

Both cortical surface reconstruction and estimation of cortical thickness were obtained with Freesurfer v4.05 (http://surfer.nmr.mgh.harvard.edu/). Manual editing was used to enhance the pial/white matter boundaries and, in turn, to obtain better estimations of cortical thickness. The analysis pipeline for cortical reconstruction and thickness estimation is summarized in Figure 1A, and extended in the Supplementary material (SM1). 


\subsection{Parcellating the neocortex with different resolution scales}

We sought to determine whether the number (or the size) of gray-matter cortical regions influenced the emergence of topological properties in cortical networks. To accomplish this goal, different cortical scales (see Figure 1B) were established starting from the original regions contained in the Desikan-Killiany atlas. This atlas divides the human neocortex into 66 standard gyral-based regions in a reliable manner (Desikan et al., 2006).

To generate a broad range of cortical scales, a backtracking algorithm was applied on the Desikan-Killiany atlas as follows. First, a seed vertex located in the periphery of an atlas region is randomly chosen. Second, the nearest vertices to the seed within that atlas region are joined until the pre-established size of the new parcel is reached. Third, if any of the remaining vertices in the atlas region becomes isolated, the new parcel is ruled out and the process starts from the first step using a different seed. This backtracking algorithm ensures that emergent cortical parcels are always built over a set of connected vertices. To further clarify these steps, a pseudocode of this parcellation algorithm can be found in Appendix A. By using this approach, we computed 23 cortical scales, each one with a different number of cortical regions and area size (Table 1). All these scales were first computed on the cortical surface of the average subject. Next, they were transformed to the subject's native space to avoid spatial mismatching of a same cortical parcel between subjects. Figure 1B shows examples of two cortical scales obtained with our backtracking algorithm (599 and 1494 cortical regions).

\subsection{Building anatomical cortical networks}

\subsubsection{Partial correlation matrices}


Graph theory implicitly assumes that two elements are "anatomically connected" if they are significantly correlated (He et al., 2007). However, high correlation values between two distant regions are likely affected by neighbour regions, which make estimations of network connectivity imprecise. To partially overcome this confounding, anatomical networks were built on the basis of partial correlations of interregional gray-matter thickness (a schematic representation of this procedure is illustrated in Figure 1C). Effects of age, gender, age-gender interaction, and mean overall cortical thickness were removed by applying a linear regression analysis across cortical regions.

In our experimental design, the number of observations (experimental subjects, $\mathrm{N}=30$ ) is smaller than the number of dependent variables (cortical regions, ranging from 66 to 1494). "Small $n$, large $p$ " experimental designs lead to inaccurate estimations of the covariance matrix caused by the elevated mean square error values derived from overfitted regression models (Peng et al., 2009).

Two recent studies have introduced alternative approaches to address this drawback (Lee et al., 2011; Huang et al., 2010), but neither of them was focused on determining small-world properties underlying cerebral networks nor did they use anatomical brain descriptors. We employed a newly developed technique based on the Ledoit-Wolf lemma to shrink the covariance estimates (Schäfer and Strimmer, 2005; Opgen-Rhein et al., 2007). Briefly, the shrinkage process performs a correction of the covariance between regions aimed at reducing the mean square error derived from the regression analysis. This method not only shows a high precision rate when controlling for false positives, but it also reduces the computation time that becomes critical in highly grained parcellation scales. Finally, the partial correlation derived from the corrected covariance matrix was calculated as: 


$$
r_{i, j}=-S_{i, j}^{-1} / \sqrt{S_{i, i}^{-1} \times S_{j, j}^{-1}}
$$

where $S_{i, j}^{-1}$ represents the $\{i, j\}$ th element of the inverted covariance matrix $S^{-1}$ computed from all possible pairs of cortical regions.

\subsubsection{Testing statistical significance}

Student's $t$ test cannot be applied to "small $n$, large $p$ " experimental designs because they result in statistical distributions with negative degrees of freedom. Instead, Monte Carlo permutation tests were used to estimate an approximated distribution derived from the shrinkage and partial correlation processes. The thickness value of each cortical scale in one particular subject was randomly reassigned to a different subject. Random partial correlations were calculated over the permutated thickness values by using the same shrinkage procedure employed with the original data. The set of resulting random $r$-values was employed as a reference distribution for statistical inference purposes. This approach provided a free distribution without a priori assumptions or a model structure fixation. We only performed 8 permutations for each cortical scale due to the high computational cost of this process (ranging from 10 seconds in the coarsest parcellation scheme to 53 days in the finest one, using Dell ${ }^{\mathrm{TM}}$ workstations with 4 Intel Xeon ${ }^{\mathrm{TM}}$ Dual Core processors, $3.2 \mathrm{GHz}$ each, 32 GB RAM, and MATLAB ${ }^{\circledR}$ v. 7.9 under Linux Centos4 X86-64 bits).

Microarray data analysis exploits gene similarity to pool permutation results before testing statistical significance (Friedman et al., 2008). We also benefit from similar cortical thickness values to generate a statistical distribution based on $r$-values derived from all pair-wise comparisons for the 8 permutations performed on each cortical scale. Thus, $p$-values were obtained by using the distribution resulting from $8 \cdot n(n-1) / 2 r$ values derived from permutated thickness values, where $n$ represents the number of 
cortical regions. We further incorporated the standard error associated to each $p$-value $\left(p_{\text {err }}\right)$. In a Monte-Carlo test, when $\mathrm{N}$ is large and $N>>N^{\prime}$, the $95 \%$ margin of error of the $p$-value is $1.96 \sqrt{p(1-p) / N^{\prime}}$ where $p$ represents the cut-off $p$-value $\left(p_{\text {cut-off }}\right)$ derived from the permutation test, $N$ denotes the number of total possible permutations, and $N^{\text {, }}$ refers to the number of permutations (Manly, 1998). We searched for the $r$-value with a $p_{\text {cut-off }}=p_{\max }-p_{\text {err }}$ with $p_{\max }=0.05$ such that the significance level of the $r$-value is $5 \%$ or lower. For instance, a cortical scale consisting of 599 regions results in 1.432 .808 permutations and $p_{\text {err }}=3.610^{-4}$ (for $p_{\max }=0.05$ ). Thus, the $p$-value used in the test is $p_{\text {cut-off }}=0.04964$. False positive correlations due to large-scale testing scenarios were corrected by using a FDR plug-in $(q$-value $<0.1)$ based on permutation methods (Hastie et al., 2001). Significant network connections within a specific cortical scale were determined by using the cut-off $r$-value obtained with Monte Carlo permutation methods. Finally, the partial correlation matrix was transformed into a binary thresholded matrix to determine topological properties of anatomical networks derived from each cortical scale.

\subsubsection{Adjacency matrix and sparsity}

In graph theoretical analysis, the percentage of nodes connected over the total possible connections is known as sparsity. This term is mathematically defined as $2 K / n(n-1)$, where $K$ represents the total number of edges (connections) and $n$ denotes the number of nodes (cortical regions) within the graph. The present study considered a wide range of sparsities to reduce bias related to the choice of cortical scale with the best topological properties. Thus, we computed a different adjacency matrix with a different sparsity level for each threshold used in the partial correlation matrix. 
The lowest sparsity boundary at which the network is fully connected can be theoretically approximated as follows (Zhu et al., 2010; Bollabas, 1985):

$$
\text { sparsity }>>\ln (n) /(n-1)
$$

where $n$ denotes the number of nodes (cortical regions). But this theoretical boundary raises the question of how high this sparsity should be. To answer this question, we searched for the lowest sparsity value (starting at 0 with a resolution of $0.1 \%$ ) in each cortical scale until the graph got fully connected. Network analyses were completed with sparsities multiple of 1 until reaching 30 . Values beyond $30 \%$ were disregarded because they provided random graphs in terms of small-world topology, and because they showed a high percentage of statistically non-significant connections in most of the cortical scales used in the present study. We further evaluated the sparsity value that yielded maximum differences in small-world properties when comparing real with random cortical networks (optimal sparsity, OS in the following).

The connectivity distribution of small-world anatomical networks was also analyzed as a function of cortical scales. The degree distribution $p(k)$ denotes the fraction of regions (nodes) with $k$ connections (edges). To decrease the noise effect, we calculated the cumulative degree distribution as $P(k)=\sum_{k^{\prime} \geq k} p\left(k^{\prime}\right)$. Typically, the cumulative degree distribution of a small-world network is fitted into different categories: scale-free, $P(k) \sim k^{-\alpha}$, exponentially decay $P(k) \sim e^{-\alpha \cdot k}$, and truncated power law $P(k) \sim k^{\alpha-1} \cdot e^{k / k_{c}}$ (Amaral et al., 2010).

\subsection{Determining small-world properties in anatomical cortical networks}

Small-world metrics were computed on the thresholded, binarized matrix of partial correlations (illustrated in Figure 1C). Information about local connectivity is provided 
by the clustering coefficient $\left(C_{p}\right)$ whereas the efficacy of long distance communication is obtained with the path length $\left(L_{P}\right)$ (Watts and Strogatz, 1998). Small-world properties were also described in terms of local $\left(E_{l o c}\right)$ and global $\left(E_{\text {glob }}\right)$ efficiency of the network. Detailed explanation of each of these small-world metrics is found elsewhere (Rubinov and Sporns, 2010; Newman, 2003; Latora and Marchiori, 2001).

Topological properties of a given network may be influenced by intrinsic features of that network, such as the number of nodes, number of connections, and degree distribution. To counteract these effects, we generated 100 random networks by using the rewiring process described by Maslov and Sneppen (2002). This algorithm preserves the number of regions, mean degree (mean number of connections considering all regions of a given network), and degree distribution (frequency distribution of connections considering all regions of a given network) as in the real network. Next, small-world properties derived from each metric and cortical scale were divided by those obtained with the above-randomized networks. As a result, we obtain a normalized clustering coefficient $\gamma_{g}=C_{p} / C_{\text {rand }}>>1$ and a normalized path length $\lambda_{g}=L_{p} / L_{\text {rand }} \approx 1$ (Watts and Strogatz, 1998), where $C_{p}$ represents the average clustering coefficient of the network, $C_{\text {rand }}$ is the average clustering coefficient of the randomly rewired networks, $L_{p}$ denotes the path length of the network, and $L_{\text {rand }}$ indicates the path length of the randomly rewired networks. The two criteria are integrated into one metric to determine the small-worldness of a specific network (Watts and Strogatz, 1998):

$$
\sigma=\gamma_{g} / \lambda_{g}
$$


Local and global efficiency descriptors are also normalized and summarized in a scalar measure of network efficiency:

$$
E_{\mathrm{lg}}=E_{l o c} \cdot E_{g l o b}
$$

The motivation of this metric is to enhance as much as possible the local efficiency keeping the global efficiency at maximum, similarly to the conditions expressed in $\gamma_{g}$ and $\lambda_{g}$. Both metrics, $\sigma$ and $E_{\mathrm{lg}}$, were used to determine small-world properties of each cortical scale employed in the present study.

\subsection{Maximizing small-world properties in anatomical cortical networks}

To determine the best trade-off between cortical resolution and small-world properties, we searched for the maximum gain in small-world properties - either $\sigma$ or $E_{\mathrm{lg}}(\mathrm{y}$-axis $)$ with the minimum number of cortical regions (x-axis) by computing the nearest distance to the superior-left corner $(\mathrm{x}=0, \mathrm{y}=1)$ of the normalized representation (see Figure 5). The shortest distance to the corner $\left(d_{c}\right)$ for each cortical scale is defined as:

$$
\sqrt{(\text { normalized number of parcels })^{2}+(1-\text { nomalized metric value })^{2}}
$$

Cortical scales showing maximum gains in small-world properties (i.e., the value of $\sigma$ and $E_{\lg }$ showing the shortest distance to corner, $d_{c}$ ) were computed not only for the optimal sparsity but also for the next four sparsities whose metric values were closer to the optimal one. As a result, we obtained 5 sparsities for each metric $\left(\sigma\right.$ and $\left.E_{\mathrm{lg}}\right)$ and cortical parcellation scheme (see Table 2 and Figure 1D, bottom panel).

Axes normalization [0-1] resulting from the relationship between the sigma-ratio and the number of cortical regions introduces changes in the shortest $d_{c}$ as a consequence of the minimum and maximum number of cortical regions considered in the analysis. 
These changes themselves could indeed modify the choice of the cortical scale. To test the robustness of our procedure, we computed the shortest $d_{c}$ after sequentially eliminating the coarsest and the finest cortical scale. Thus, axes normalization was sequentially computed each time with 2 n less cortical scales (n ranging from 0 to 8 ). Consequently, $d_{c}$ was calculated for $24,22,20,18,16,14,12,10$ and 8 cortical scales. Results from this sequential analysis are shown in Table 2 . The cortical scale with the most efficient topology was defined as the one showing the most consistent $d_{c}$.

\subsection{Determining resilience to attacks in different cortical scales}

To investigate variations in nodal features as a function of cortical resolution, the degree and betweenness of nodes were computed in each cortical scale. The degree $K_{i}$ of node $i$ refers to the number of connections reaching that specific node, whereas betweenness $B_{i}$ is defined as the number of shortest paths between any two nodes that pass through the node $i$ (Freeman, 1977). The normalized betweenness was computed as $b_{i}=B_{i} /\langle B\rangle$, where $\langle B\rangle$ denotes the average betweenness of the network (He et al., 2008), and was calculated with the MatlabBGL package (http://www.stanford.edu/ dgleich/ programs/matlab bgl/). Network hubs were identified as the cortical regions with high degree and high betweenness.

The resilience of anatomical cortical networks to targeted attacks was further evaluated by removing the hubs one by one in rank order of decreasing degree (this strategy was also performed by using a decreasing betweenness criterion), whereas random failures were designed by eliminating nodes randomly. This process was then repeated, incrementally eliminating $5 \%$ of the network nodes. A fraction of the nodes was sequentially removed before recalculating the path length and the size of the largest connected component denoting the largest proportion of nodes connected to each other. 
We compared nodal characteristics and network resilience between the cortical scale resulting from the application of our procedure and those either based on the DesikanKilliany atlas or in the backtraking algorithm used in the present study.

\section{Results}

\subsection{Effects of cortical scale and sparsity on small-world properties}

Topological attributes and sparsity for each cortical scale are shown in Table 1. The theoretical sparsity defined by formula (2) predicts the lowest bound of a sparsity range at which the network becomes fully connected for each cortical scheme. As expected, the minimum empirical sparsity was in most cases higher than that provided by the theoretical sparsity. Note also the opposite result obtained with 108 cortical regions.

The optimal sparsity differed between small-world metrics ( $\sigma$ and $E_{\mathrm{lg}}$ ), and it decreased with finer cortical scales (Table 1). All interregional correlations were significant at the optimal sparsity with the two metrics $(p<0.05$; FDR corrected). Interestingly, the fraction of significant correlations decreased with finer cortical scales, ranging from $50 \%$ in the coarsest scale to $4 \%$ in the finest scale, suggesting that the statistical power of small-world properties decreases with highly grained scales.

Previous studies have also examined small-world properties of cerebral networks for a broad range of sparsities (e.g., Bassett et al., 2008; Zhu et al., 2010). In line with these studies, our results show an exponential decay of network efficiency ( $\sigma$ metric) with the increase of sparsity (Figure 2A). This relationship was statistically confirmed for all cortical scales by applying a linear regression analysis over the natural logarithm of these two variables $\left(\mathrm{R}^{2}\right.$ ranged from 0.95 to 1 , and $p$-values from $10^{-32}$ to $10^{-13}$ after Bonferroni correction; Figure 3A). Note that maximum $\sigma$ values were frequently 
associated with sparsities lower than or equal to 8 (Figure $2 \mathrm{~A}$ and Table 1). As predicted by the small-world theory, enhancements of network efficiency $(\sigma)$ were mainly due to increased local connectivity $\left(C_{p}\right)$ rather than to superior ability to transfer information at a global level $\left(L_{P}\right)$ as illustrated in Figures 2B and 2C, respectively.

Results derived from the $\sigma$ metric might lead to erroneous inferences about the optimal sparsity. One may indeed conclude that the optimal sparsity corresponds to the first value that fully connects the network. However, this reasoning seems inappropriate when measuring the capacity of the network to continue operating properly in the presence of a failure (fault-tolerance or robustness) as revealed by $E_{l g}$, and more particularly by $E_{l o c}$. Accordingly, $E_{l g}$ and $E_{l o c}$ values increased with sparsity up to a point and then they decreased rapidly (Figures $2 \mathrm{D}$ and $2 \mathrm{E}$, respectively). Given that $E_{\text {glob }}$ varied slightly with sparsity (Figure $2 \mathrm{~F}$ ), we assume that the relationship between $E_{l g}$ and sparsity in anatomical cortical networks is mainly determined by local rather than global topological properties. The quadratic relationship between $E_{l g}$ and sparsity was statistically confirmed for all cortical scales by using polynomial linear regression analysis $\left(2^{\text {nd }}\right.$ degree) performed over the natural logarithm of these two variables $\left(\mathrm{R}^{2}\right.$ ranged from 0.81 to 0.99 , and $p$-values from $10^{-28}$ to $10^{-7}$ after Bonferroni correction; Figure 3B).

Unlike local properties, global efficiency approximated to the maximum with sparsities above 10 for the majority of cortical scales (Figures 2C and 2F). Global efficiency of finer cortical scales deviated from 1 (the maximum) more frequently when compared to coarser cortical scales at sparsities lower than 8 . This suggests that global properties of anatomical cortical networks are less comparable with a random-like network at finegrained cortical scales (Bassett et al., 2008). The fact that global properties (but not the 
local ones) impair with finer scales has been recently confirmed by using cerebral diffusion tensor images (Zalesky et al., 2010). This finding together with the elevated computational costs associated with fine-grained cortical scales adds support against using highly parcellated cortical schemes for assessing small-world properties in anatomical cortical networks.

Figure 4 shows, for different cortical scales, the fitting of these functions over the cumulative node degree distribution. In every case the best fitting was provided by the truncated power law, which reduces the number of cortical hubs with a degree greater than the cut-off value $\left(k_{c}\right)$.

\subsection{Trade-off between cortical resolution and small-world properties}

We tried to determine the best scenario to maximally preserve small-world properties in anatomical cortical networks. To this aim, the nearest distance to the superior-left corner $\left(d_{c}\right)$ was computed along the normalized axes with two different small-world metrics ( $\sigma$ and $E_{l g}$ ). Normalization is required to compare network efficiency across different cortical scales, since both coordinate axes (number of regions and sigma ratios) have different orders of magnitude and units. To determine whether the weighting introduced by the normalization process affected the choice of the cortical scale, axes normalization was performed in a varying range of cortical scales, which implicitly varies the original weight. Results from this analysis revealed that reducing sequentially the range of regions (min-max) did not affect critically the selected cortical scale (see Table 2). 41 of 45 estimations obtained with the $\sigma$ metric at the 5 best sparsities suggests that the cortical scale comprising 599 regions represents the best trade-off between small-worldness and resolution of the cortical scale. The selected cortical scale varied slightly for the $E_{\lg }$ metric (ranging from 541 to 599 regions) when computed with the 5 
best sparsities. More specifically, it showed a bimodal distribution comprising 39 of 45 estimations (571 and 541 peaked 20 and 19 times, respectively). When results derived from both metrics were combined, cortical scales resulting from the above mentioned trade-off ranged from 541 to 599 regions. No significant differences were found between small-world properties obtained with these cortical scales after Bonferroni correction, either for $\sigma$ or $E_{l g}$. However, small-world properties, at least for $E_{l g}$, were significantly higher at the cortical scale of 599 regions when compared with coarser scales $(\mathrm{p}<0.02$ after Bonferroni correction). In the case of the $\sigma$ metric, statistical significance was only reached for cortical scales below 198 regions $\left(\mathrm{p}<10^{-4}\right.$ after Bonferroni correction). In summary, our results suggest that 540-600 gray-matter regions seems to provide a satisfactory trade-off between small-world properties and the number of cortical regions considered in the scale, at least for the population evaluated in the present study.

We further assessed changes in network efficiency for the five best sparsities obtained for each cortical scale (Figure 5). Results derived from the $E_{l g}$ metric (Figure 5B) were more stable across cortical scales when compared with those obtained with the $\sigma$ metric (Figure 5A). This analysis also revealed that differences in network efficiency decreased across cortical scales with larger deviations from the optimal sparsity, suggesting a loss of sensibility to detect topological changes with less favourable sparsities.

\subsection{Measuring network robustness in different anatomical cortical scales}

Nodal characteristics and network resilience were also compared between the selected cortical scale (599 regions) and other representative schemes (Desikan-Killiany atlas 66, 108 and 1494 regions) for a sparsity of $8 \%$ (the lowest sparsity value common to the four selected scales). Table 3 shows the anatomical location of the top 7 hubs whose 
degree and betweenness were 1.5 standard deviations over the mean. Figure 6 illustrates the largest connected component (Figure 6A and 6B, upper row) and the path length (Figure 6A and 6B, bottom row) for each one of these three cortical scales (solid line) compared with that derived from the selected cortical scale providing the best trade-off (dashed line), as a function of the fraction of nodes removed in random and targeted attacks. The latter cortical scale was more resilient to targeted attacks than that derived from either the Desikan-Killiany atlas (66 regions) or the cortical scheme with 108 regions. More specifically, a substantial reduction of the largest component was found after removing $40 \%$ of the top hubs in the network derived from the Desikan-Killiany atlas. It was required to eliminate $65 \%$ of top hubs to get $\sim 24 \%$ reduction of the largest component in the cortical scale comprising 108 regions. Both the selected cortical scale (599 regions) and the finest one included in the present study (1494 regions) showed similar resilience to targeted attacks (Figure 6A). Only the atlas-based parcellation showed vulnerability to random attacks with the largest component. Targeted attacks further resulted in increased path length before fragmentation of both the atlas-based network and the cortical network comprising 108 regions (Figure 6B). The path length increased threefold when $60 \%$ of nodes were removed from the network of 108 regions, and it increased twofold when targeted attacks were restricted to $35 \%$ of nodes in the atlas-based network. It is worth noting the robustness to targeted attack of the selected cortical scale (599 regions) compared with the one derived from the Desikan-Killiany atlas and from the cortical scale of 108 regions. Similar results were obtained when cortical hubs were ranked by betweenness instead of considering their node degree. Resilience to random and targeted attack of the selected cortical scale was similar to that resulting from the finest cortical scale employed in this study (Figure 6, right column). 


\section{Discussion}

Graph theory has borrowed the conservation laws formulated by Ramon y Cajal (19091911) to shed light on how cortical networks remain highly efficient with a low wiring cost. In agreement with these principles of neuronal organization, large-scale cortical networks have shown a small-world organization characterized by cohesive neighbourhoods and short path-lengths between remote regions. Although cortical networks represent the anatomical substrate of most of the cognitive processes in mammals, few studies to date have described the topological organization of human anatomical cortical networks by using neuroimaging techniques (Fan et al., 2010; Gong et al., 2009; He et al., 2007). Recent evidence support the notion that the topology of anatomical cerebral networks varies considerably as a function of the scale resolution employed (Zalesky et al., 2010; Sanabria-Diaz et al., 2010). The present work adds support to that hypothesis, and it further provides a novel methodological framework to identify cortical scales showing the best trade-off between small-world properties and cortical resolution by using thickness measurements as a measure of anatomical connectivity.

We found that highly grained cortical scales showed enhanced local connectivity (as revealed by the clustering coefficient), local efficiency (showed by the $E_{l o c}$ metric), and small-worldness (provided by $\sigma$ and $E_{l g}$ metrics) together with a lower vulnerability to targeted attacks. In agreement with previous reports (Zalesky et al., 2010), we further found increased path length and decreased global efficiency with finer cortical scales. Contrary to the approach followed by Zalesky and colleagues (2010), the trade-off between cortical scales and small-world properties was reached by keeping constant the sparsity. 
Our results suggest that determining the best trade-off between the resolution of the cortical scale and the level of small-worldness in anatomical cortical networks requires from assessing a broad range of sparsity values (e.g., Supekar et al., 2009). We indeed found that the optimal sparsity not only varied with the cortical scale but also with the small-world metric employed. Contrary to previous studies that evaluated network topology at sparsities above 12\% (Bassett et al., 2008; Liu et al., 2008; Lynall et al., 2010; Sanabria-Diaz et al., 2010), our results highlight the importance of including lower sparsity values in large-scale network analysis. First, because the lowest sparsity at which the network becomes fully connected and the optimal sparsity for the $\sigma$ metric are identical. And, secondly, because the optimal sparsity for the $E_{l g}$ metric was below $8 \%$ with most of the cortical scales considered in the present study. Taken collectively, our findings add support to previous studies that also reported increased small-world properties with low sparsity values (Bassett et al., 2008; Lv et al., 2010; Zhu et al., 2010).

The main rationale of this work was to establish a satisfactory trade-off between smallworld attributes derived from anatomical cortical networks and the number/size of graymatter regions included in the cortical scale. Overall, our results suggest that the best topological trade-off can be determined in anatomical cortical networks by using a number of gray-matter regions ranging between 540 and 599, which correspond to areas spanning between 250 and $275 \mathrm{~mm}^{2}$, respectively. We have further confirmed that our approach is independent of (i) the number of cortical scales included in the analysis; (ii) how far the sparsity deviates from the optimal one; and (iii) the metric employed to describe small-worldness. In addition, the cortical scale selected (599 regions) was more resilient to targeted attacks when compared with coarser cortical scales derived from either the Desikan-Killiany atlas or random criteria (108 cortical regions). In particular, 
we found that the cortical scale resulting from the trade-off analysis only became fully disconnected when $95 \%$ of top-hubs were removed; whereas a targeted attack on $45 \%$ and $65 \%$ of hubs was enough to disconnect both the Desikan-Killiany and the cortical network comprising 108 regions, respectively. But, most importantly, the cortical scale selected showed similar resilience than the finest one (1494 regions) used in the present study.

Our procedure works well with concave functions (derived from the relationship between $\sigma$ and the number of cortical regions), but it seems inappropriate when this relationship fits to linear or exponential (convex) functions. An exponential growth of small-world properties with the cortical scale results in similar distance to corner $\left(d_{c}\right)$ for both the coarsest and finest scale, which would impede the use of this selection criterion. On the other hand, criteria derived from a linear function would select the average between the coarsest and finest cortical scale as the best one, which might not coincide with the best trade-off between small-world properties and cortical resolution. In addition, both types of functions (linear and exponential) mathematically fail to define an optimal point and therefore they would be highly dependent on the first and last cortical scales employed in the study. In line with our results, Zalesky et al. (2010) showed a similar relationship between $\sigma$ and the number of brain regions by using cerebral tractography, pointing to this behaviour as a universal property of anatomical cerebral networks.

In the present study, criteria considered to determine the trade-off between small-world properties and resolution of the cortical scale were based exclusively on topological properties of the underlying anatomical network. However, these criteria might not be adequate in those studies motivated by a priori or neurobiological hypotheses. Summarizing, the choice of a specific cortical scale may not apply to all experimental 
settings. It will strongly depend on the nature of the study (exploratory versus hypothesis-driven) and on the underlying hypothesis (topological versus biological). We speculate that cerebral scenarios maximizing small-world properties should enhance the identification of changes in topological features caused by brain dysfunction or lesions, but this hypothesis needs to be experimentally supported with further experiments.

Anatomical networks based on brain atlases are primarily modeling integrity of the callosal system connecting both cerebral hemispheres as well as the fascicles connecting the lobes of one hemisphere with each other (fiber length ranging from 30 to $100 \mathrm{~mm}$ ), but they largely neglect the U-fiber system that connects cortical gray matter (fiber length ranging from 3 to $30 \mathrm{~mm}$, average length of $15 \mathrm{~mm}$ ) (Schüz and Braitenberg, 2002). Our approach allows us to model the cortical topology preserving relatively constant the fiber length included in each cortical module. In order to model the neural organization intrinsic to the U-fiber system, we assumed that (i) the upper limit of the axon length is $30 \mathrm{~mm}$, (ii) these fibers approximately follow a rectilinear path, and (iii) cortical regions have a circular shape (as a result of the parcellation procedure used in the present study). Following these conditions, the region size required to characterize the U-fiber system must be smaller than $707 \mathrm{~mm}^{2}$, meaning that the cortical scale should contain about 200 gray-matter regions. Therefore, we speculate that the U-fiber system may be responsible for the drastic increase of the local efficiency associated with highly grained cortical scales, including those revealing a superior balance between small-world properties and the resolution of the cortical scale (spanning between 540 and 600 gray-matter regions).

The present study poses some limitations that should be noted: (i) we infer anatomical connectivity from a linear regression model applied to cortical thickness measurements. This approach implicitly assumes anatomical relationships between cortical regions (He 
et al., 2007) rather than anatomical connections between them. Although the nature of this anatomical correlation is poorly understood, previous studies have found correlations between different cortical descriptors (thickness or volume) and trophic influence between regions (Ferrer et al., 1995), genetic (Thompson et al., 2001) and environmental factors (Mechelli et al., 2005); (ii) topological features of anatomical cortical networks were obtained from the entire population by using regression analysis impeding to draw conclusions from individual subjects (scheme used in fMRI and EEG/MEG studies, see Hayasaka et al., 2010; Stam et al., 2009). This issue becomes especially problematic in clinical settings; (iii) the choice of the best sparsity neglects the bias introduced by lower connection densities; (iv) significant connections comprising our anatomical cortical networks were obtained from binary graphs. Weighted graphs could have alternatively been used although they seem more appropriate to establish functional connectivity patterns (Guye et al., 2010); (iv) topological properties of cortical scales finer than 1494 cortical regions were not explored in this study due to excessively longer computation time; and (v) criteria to select the cortical scale resulting from the trade-off between small-world properties and cortical resolution were exclusively based on topological considerations instead of on biological organization of the human neocortex. As a consequence, hypothesis-driven studies might not benefit from the choice of a cortical scale since it could enter in conflict with the neurobiological hypothesis under study.

\section{Conclusions}

The present work systematically assesses the impact of the cortical scale on the smallworld properties derived from anatomical cortical networks. More importantly, we 
provided a novel framework to establish an effective trade-off between small-world attributes of anatomical cortical networks and number/size of gray-matter regions. Although we showed that anatomical cortical networks comprised by nodes of $250 \mathrm{~mm}^{2}$ and sparsities below $8 \%$ reveal enhanced manifestations of the small-world architecture, the use of this cortical scale will critically depend on the nature of the study (exploratory versus hypothesis-driven) and on the underlying hypothesis (topological versus biological). Thus, if one is mainly interested in modelling the anatomical connectivity governed by the long-range fibre system, coarser scales may be more appropriate to grasp the network topology of structural networks. But if the focus of interest is the U-fibre system connecting neuronal populations within the same cortical module, then finer cortical scales should be considered. 


\section{Appendix A}

Pseudocode of the parcellation algorithm

for $i=1,2 \ldots 66$

consider $\mathrm{i}$ as one atlas region fully uncovered

while i region is not fully covered

create a new empty parcel

add to the new parcel a random seed vertex belonged to the uncovered i region

while the parcel doesn't reach the desired area

add to the parcel the nearest uncovered vertex

end while

if still remain uncovered vertices of the atlas region and they are not connected undo the new parcel

else

establish the new parcel as covered area

end if

end while

end for 


\section{Acknowledgments}

This study was supported by grants from the Spanish Ministry of Science and Innovation (SAF2008-03300; SAF2011-25463) and the Regional Ministry of Innovation, Science and Enterprise, Junta de Andalucia (P09-CTS-4604) given to JLC. 


\section{References}

Amaral, L.A., Scala, A., Barthelemy, M., Stanley, H.E. 2000. Classes of small-world networks. Proc. Natl. Acad. Sci. USA 97, 11149-11152.

Bassett, D.S., Bullmore, E., Verchinski, B.A., Mattay, V.S., Weinberger, D.R., MeyerLindenberg A. 2008. Hierarchical organization of human cortical networks in health and schizophrenia. J. Neurosci. 28, 9239-9248.

Bassett, D.S., Bullmore, E. 2006. Small-world brain networks. Neuroscientist 6, 512523.

Bollabas, B. 1985. Random graphs. London: Academic Press. 447 p.

Desikan, R.S., Ségonne, F., Fischl, B., Quinn, B.T., Dickerson, B.C, Blacker, D., Buckner, R.L., Dale, A.M., Maguire, R.P., Hyman, B.T., Albert, M.S., Killiany, R.J. 2006. An automated labeling system for subdividing the human cerebral cortex on MRI scans into gyral based regions of interest. Neuroimage 31, 968-980.

Douglas, R.J., Martin, K.A. 2004. Neuronal circuits of the neocortex. Annu. Rev. Neurosci. 27, 419-451.

Fair, D.A., Cohen, A.L., Power, J.D., Dosenbach, N.U.F., Church, J.A., Miezin, F.M., Schlaggar, B.L., Petersen, S.E. 2009. Functional brain networks develop from a "local to distributed" organization. PLoS Comp. Biol. 5, e100381.

Fan, Y., Shi, F., Smith, J.K., Lin, W., Gilmore, J.H., Shen, D. 2010. Brain anatomical networks in early human brain development. Neuroimage 54, 1862-1871. 
Ferrer, I., Blanco, R., Carulla, M., Condom, M., Alcantara, S., Olive, M., Planas, A. 1995. Transforming growth factor-alpha immunoreactivity in the developing adult brain. Neuroscience 66, 189-199.

Freeman, L.C. 1977. Set of measures of centrality based on betweenness. Sociometry $40,35-41$

Friedman, J., Hastie, T., Tibshirani, R. 2008. High-Dimensional Problems: p >> N. In: The elements of statistical learning: Data mining, inference and prediction. New York: Springer, pp 697-754.

Gong, G., He, Y., Concha, L., Lebel, C., Gross, D.W., Evans, A.C., Beaulieu, C. 2009. Mapping anatomical connectivity patterns of human cerebral cortex using in vivo diffusion tensor imaging tractography. Cereb. Cortex 19, 524-536.

Guye, M., Bettus, G., Bartolomei, F., Cozzone, P.J. 2010. Graph theoretical analysis of structural and functional connectivity MRI in normal and pathological brain networks. MAGMA 23, 409-421.

Hagmann, P., Cammoun, L., Gigandet, X., Meuli, R., Honey, C.J., Wedeen, V.J., Sporns, O. 2008. Mapping the structural core of human cerebral cortex. PLoS Biol. 6, e159.

Hastie, T., Tibshirani, R., Friedman, J. 2001. The elements of statistical learning. New York: Springer. $552 \mathrm{p}$.

Hayasaka, S., Laurienti, P.J. 2010. Comparison of characteristics between region-and voxel-based network analyses in resting-state fMRI data. Neuroimage 50, 499-508. 
He, Y., Dagher, A., Chen, Z., Charil, A., Zijdenbos, A., Worsley, K., Evans, A. 2009. Impaired small-world efficiency in structural cortical networks in multiple sclerosis associated with white matter lesion load. Brain 132, 3366-3379.

He, Y., Chen, Z.J., Evans, A.C. 2008. Structural insights into aberrant topological patterns of large-scale cortical networks in Alzheimer's disease. J. Neurosci. 28, 47564766.

He, Y., Chen, Z.J., Evans, A.C. 2007. Small-world anatomical networks in the human brain revealed by cortical thickness from MRI. Cereb. Cortex 17, 2407-2419.

Huang, S., Li, J., Sun, L., Ye, J., Fleisher, A., Wu, T., Chen, K., Reiman, E.; Alzheimer's Disease NeuroImaging Initiative. 2010. Learning brain connectivity of alzheimers disease by sparse inverse covariance estimation. Neuroimage 50, 935-949.

Krubitzer, L. 2007. The magnificent compromise: cortical field evolution in mammals. Neuron 56, 201-208.

Latora, V. Marchiori, M. 2001. Efficient behaviour of small-world networks. Phys. Rev. Lett. 87, 198701.

Lee, H., Lee, D.S., Kang, H., Kim, B.N., Chung, M.K. 2011. Sparse brain network using penalized linear regression. Proc. SPIE Med. Imag., 7965-7942.

Liao, W., Ding, J., Marinazzo, D., Xu, Q., Wang, Z., Yuan, C., Zhang, Z., Lu, G., Chen, H. 2011. Small-world directed networks in the human brain: Multivariate Granger causality analysis of resting-state fMRI. Neuroimage 54, 2683-2694.

Liu, Y., Liang, M., Zhou, Y., He, Y., Hao, Y., Song, M., Yu, C., Liu, H., Liu, Z., Jiang, T. 2008. Disrupted small-world networks in schizophrenia. Brain 131, 945-961. 
Lv, B., Li, J., He, H., Li, M., Zhao, M., Ai, L., Yan, F., Xian, J., Wang, Z. 2010. Gender consistency and difference in healthy adults revealed by cortical thickness. Neuroimage $53,373-382$.

Lynall, M.E., Bassett, D.S., Kerwin, R., McKenna, P.J., Kitzbichler, M., Muller, U., Bullmore, E. 2010. Functional connectivity and brain networks in schizophrenia. J. Neurosci. 30, 9477-9487.

Manly, D.F.J. 1998. Randomization, bootstrap and Monte Carlo methods in biology. London: Chapman \& Hall. 480 p.

Mantegna, R.N., Stanley H.E. 2000. An introduction to econophysics: correlations and complexity in finance. Cambridge, MA: Cambridge University Press. 164 p.

Maslov, S., Sneppen, K. 2002. Specificity and stability in topology of protein networks. Science 296, 910-913.

Mechelli, A., Friston, K.J., Frackowiak, R.S., Price, C.J. 2005. Structural covariance in the human cortex. J. Neurosci. 25, 8303-8310.

Newman, M.E.J. 2003. The structure and function of complex networks. SIAM Rev. Soc. Ind. Appl. Math. 45, 167-256.

Opgen-Rhein, R., Strimmer, K. 2007. Accurate ranking of differentially expressed genes by a distribution-free shrinkage approach. Stat. Appl. Genet. Mol. Biol. 6, 9.

Peng, J., Wang, P., Zhou, N., Zhu, J. 2009. Partial correlation estimation by joint sparse regression models. J Am Stat Assoc 104, 735-746. 
Ramon y Cajal, S. 1909-1911. Histologie du système nerveux de l'homme et des vertébrés. Paris: Maloine.

Rubinov, M., Sporns, O. 2010. Complex network measures of brain connectivity: Uses and interpretations. Neuroimage 52, 1059-1069.

Sanabria-Diaz, G., Melie-Garcia, L., Iturria-Medina, Y., Aleman-Gomez, Y., Hernandez-Gonzalez, G., Valdés-Urrutia, L., Galan, L., Valdes-Sosa, P. 2010. Surface area and cortical thickness descriptors reveal different attributes of the structural human brain networks. Neuroimage 50, 1497-1510.

Schäfer, J., Strimmer, K. 2005. A shrinkage approach to large-scale covariance matrix estimation and implications for functional genomics. Stat. Appl. Genet. Mol. Biol. 4, 32.

Schüz, A., Braitenberg, V. 2002. The human cortical white matter: quantitative aspects of cortico-cortical long-range connectivity. In: Schüz A, Miller R editors. Cortical areas: unity and diversity. London: CRC Press, pp 377-385.

Shu, N., Liu, Y., Li, J., Li, Y., Yu, C., Jiang, T. 2009. Altered anatomical network in early blindness revealed by diffusion tensor tractography. PLoS One 4, e7228.

Stam, C.J., de Haan, W., Daffertshofer, A., Jones, B.F., Manshanden, I., van Cappellen van Walsum, A.M., Montez, T., Verbunt, J.P., de Munck, J.C., van Dijk, B.W., Berendse, H.W., Scheltens, P. 2009. Graph theoretical analysis of magnetoencephalographic functional connectivity in Alzheimer's disease. Brain 132, 213-224. 
Supekar, K., Musen, M., Menon, V. 2009. Development of large-scale functional brain networks in children. PLoS Biol. 7, e1000157.

Thompson, P.M., Cannon, T.D., Narr, K.L., van Erp, T., Poutanen, V.P., Huttunen, M., Lonnqvist, J., Standertskjold-Nordenstam, C.G., Kaprio, J., Khaledy, M., Dail, R., Zoumalan, C.I., Toga, A.W. 2001. Genetic influences on brain structure. Nat. Neurosci. $4,1253-1258$

Tian, L., Wang, J., Yan, C., He, Y. 2010. Hemisphere- and gender-related differences in small-world brain networks: A resting-state functional MRI study. Neuroimage 54, 191202.

Travers, J., Milgram, S. 1969. An experimental study of the small world problem. Sociometry $32,425-443$.

Wang, J., Wang, L., Zang, Y., Yang, H., Tang, H., Gong, Q., Chen, Z., Zhu, C., He, Y. 2009. Parcellation-dependent small-world brain functional networks: a resting-state fMRI study. Hum. Brain Mapp. 30, 1511-1523.

Watts, D.J., Strogatz, S.H. 1998. Collective dynamics of "small-world" networks. Nature 393, 440-442.

Zalesky, A., Fornito, A., Harding, I.H., Cocchi, L., Yucel, M., Pantelis, C., Bullmore, E.T. 2010. Whole-brain anatomical networks: does the choice of nodes matter? Neuroimage 50, 970-983.

Zhu, W., Wen, W., He, Y., Xia, A., Anstey, K.J., Sachdev, P. Changing topological patterns in normal aging using large-scale structural networks. Neurobiol. Aging. In press. 


\section{Figure legends}

Figure 1. Schematic representation of the analysis pipeline to determine the best tradeoff between the level of small-worldness and the resolution of the scale in anatomical cortical networks. A. Cortical thickness estimation. Individual cortical thickness maps were obtained from previously segmented T1-MR images. These thickness maps were averaged to create the template on which different cortical scales were determined. B. Obtaining cortical scales. Cortical scales used in the current study were originally derived from the Desikan-Killiany atlas (Desikan et al., 2006). This panel also depicts cortical scales comprising 599 and 1494 regions. C. Building cortical networks. Partial correlations of interregional thickness across subjects were computed for each cortical scale (adjacency matrices), and then they were binarized by using different sparsities (binarized matrices). Results of binarized matrices were displayed on the pial surface showing the anatomical cortical network for a given sparsity (right column). D. Enhancing network properties. Top panel illustrates results obtained with two smallworld metrics $\left(\sigma\right.$ and $\left.E_{l g}\right)$ calculated over a wide range of sparsities (1-30\%) for the 24 cortical scales used in the present study. Bottom panel displays small-world properties for all cortical scales derived from the five best sparsities. Yellow circles correspond to the cortical scale resulting from the best trade-off between small-world properties and resolution of the cortical scale (599 regions) obtained with the method based on the shortest distance to corner $\left(d_{c}\right)$.

Figure 2. Small-world properties as a function of sparsity for each cortical scale. Warm colors correspond to highly-grained cortical scales whereas cool colors refer to coarser cortical scales. Color-filled circles in each scale establishes the boundary between significant and non-significant sparsities ( $p<0.05$ FDR-corrected). A. Changes in the $\sigma$ 
metric as a function of sparsity for every cortical scale. B. Clustering coefficient (normalized with 100 random networks), this ratio $C_{p} / C_{\text {rand }}$ shows similar shape as the $\sigma$ value because local properties are prevalent in small-world networks. C. Variations in the path length $\left(L_{p}\right)$ as a function of sparsity for each cortical scale. Note that although most values are close to 1 (following the Watt and Strogatz's criterion), the finer the cortical scale the higher the $L_{p}$ for lower sparsities. D. Results obtained with the $E_{l g}$ metric, an alternative metric to $\sigma$, also suggest an increase of small-world properties with finer cortical scales. E. The local efficiency index $\left(E_{l o c}\right)$, as with $C_{p}$, enhances local connectivity in finer cortical scales and sparser networks. F. Note that global efficiency $\left(E_{g l o b}\right)$ is slightly decreased in finer cortical scales.

Figure 3. Relationships between small-world properties and sparsity. A. $\sigma$ shows a linear fit over the sparsity. B. $E_{l g}$ as a function of sparsity was fitted using a $2^{\text {nd }}$ degree polynomial. Results obtained suggest significant relationships between the two smallworld metrics and sparsity.

Figure 4. Degree distribution of cortical scales comprising 66, 108, 599, and 1494 regions. Thin solid lines represent the best fit using a scale-free $\left(P(k) \sim k^{-\alpha}\right)$ distribution, dashed lines refer to the exponential law $\left(P(k) \sim e^{-\alpha \cdot k}\right)$, and thicker solid lines represent the fit to truncated power law $\left(P(k) \sim k^{\alpha-1} \cdot e^{k / k_{c}}\right)$. The best matching was provided by a truncated power law distribution for the four cortical scales, as reported in previous neuroimaging small-world studies (Gong et al., 2009; Liao et al., 2010). CD = cumulative distribution. 
Figure 5. Small-world properties as a function of the range of cortical regions for the five best sparsities. The thicker solid line represents the best possible value of $\sigma(\mathbf{A})$ and $E_{l g}(\mathbf{B})$ regardless of sparsity. Remaining lines correspond to ranked sparsity values (optimal sparsity (OS), OS-1, OS-2, OS-3 and OS-4). The shortest distance to corner $\left(d_{c}\right)$ shows the cortical scale nearest to the left-superior corner in the normalized axis. The $d_{c}$ criteria points to 599 cortical regions as the best trade-off between cortical the level of small-worldness and the scale resolution in the five cases considered for $\sigma$. The best trade-off ranged between 541 and 571 cortical regions determined by $E_{l g}$ (for the sake of clarity, only $d_{c}$ corresponding to the first optimal sparsity was represented).

Figure 6. Determining resilience of anatomical cortical networks to random and targeted attack. Solid lines represent the robustness of cortical scales comprising 66, 108 and 1494 regions, and dashed lines correspond to the cortical scale resulting from the best trade-off between small-world properties and cortical resolution (599 regions). Network resilience to random and targeted attack was always computed for a sparsity of 8\% A. Network resilience to progressive elimination of top hubs within the cortical network. Plots in the top panel show the size of the largest component. Deviation from the diagonal indicates that the cortical network was segregated into various components. Plots in the bottom panel indicate that although the cortical network maintains a unique component after removing the first nodes, an increase of its average path length is maintained. Differences become more evident when the selected cortical scale was compared with coarser cortical scales. B. Resilience of cortical networks after a random elimination of nodes. Both the largest component (top panel) and the path length (bottom panel) suggest that the improvement in robustness observed in finer cortical scales is weaker in random than in targeted attack. 
Table 1. Small-world properties across different cortical scales.

\begin{tabular}{|c|c|c|c|c|c|c|}
\hline$\#$ areas & $\begin{array}{l}\text { Area } \\
\left(\mathrm{mm}^{2}\right)\end{array}$ & $\begin{array}{l}\text { Minimum } \\
\text { theoretical } \\
\text { sparsity } \gg>\end{array}$ & $\begin{array}{c}\text { Minimum } \\
\text { empirical } \\
\text { sparsity }\end{array}$ & $\begin{array}{l}\text { Maximum } \\
\text { significant } \\
\text { sparsity }\end{array}$ & $\begin{array}{l}\text { Optimal } \\
\text { sparsity } \\
(\operatorname{Max} . \sigma)\end{array}$ & $\begin{array}{c}\text { Optimal } \\
\text { sparsity } \\
\text { (Max. Elg) }\end{array}$ \\
\hline 66 & variable & 6.45 & 7.13 & 50 & $8.01(2.38)$ & $13(1.23)$ \\
\hline 108 & 1600 & 4.38 & 4.10 & 47 & $4.10(4.09)$ & $11(1.37)$ \\
\hline 136 & 1200 & 3.64 & 5.73 & 45 & $5.73(3.03)$ & $12(1.48)$ \\
\hline 157 & 1000 & 3.24 & 4.74 & 45 & $4.74(3.31)$ & $12(1.53)$ \\
\hline 198 & 800 & 2.68 & 4.27 & 42 & $4.27(3.54)$ & $10(1.60)$ \\
\hline 229 & 660 & 2.38 & 4.72 & 40 & $5.00(3.41)$ & $7(1.65)$ \\
\hline 347 & 431 & 1.69 & 3.74 & 33 & $4.00(3.84)$ & $7(1.85)$ \\
\hline 376 & 400 & 1.58 & 2.85 & 31 & $3.00(4.58)$ & 8 (1.89) \\
\hline 496 & 300 & 1.25 & 2.88 & 25 & $2.88(4.60)$ & $7(2.03)$ \\
\hline 541 & 275 & 1.17 & 2.55 & 23 & $2.55(5.30)$ & $6(2.08)$ \\
\hline 571 & 262 & 1.11 & 2.04 & 22 & $2.04(6.27)$ & $6(2.11)$ \\
\hline 599 & 250 & 1.07 & 1.70 & 21 & $1.69(7.14)$ & $6(2.12)$ \\
\hline 625 & 240 & 1.03 & 1.78 & 20 & $1.78(6.25)$ & $6(2.13)$ \\
\hline 644 & 231 & 1.01 & 2.03 & 20 & $2.03(5.99)$ & $6(2.17)$ \\
\hline 676 & 222 & 0.97 & 1.52 & 18 & $1.52(7.60)$ & $6(2.18)$ \\
\hline 808 & 185 & 0.83 & 2.46 & 15 & $2.46(5.32)$ & $5(2.26)$ \\
\hline 849 & 177 & 0.8 & 2.46 & 14 & $2.46(5.39)$ & $5(2.30)$ \\
\hline 879 & 170 & 0.77 & 1.36 & 13 & $1.36(7.94)$ & $5(2.35)$ \\
\hline 951 & 157 & 0.72 & 1.44 & 11 & $1.44(7.85)$ & $5(2.39)$ \\
\hline 990 & 151 & 0.7 & 1.53 & 11 & $1.53(7.49)$ & $5(2.44)$ \\
\hline 1031 & 145 & 0.67 & 2.12 & 10 & $2.12(6.12)$ & $5(2.46)$ \\
\hline 1243 & 120 & 0.57 & 1.36 & 7 & $1.36(8.16)$ & $4(2.58)$ \\
\hline 1357 & 110 & 0.53 & 1.28 & 5 & $1.28(8.82)$ & $4(2.65)$ \\
\hline 1494 & 100 & 0.49 & 1.19 & 4 & $1.19(9.14)$ & $4(2.70)$ \\
\hline
\end{tabular}

Note that the atlas-based cortical scale (66 regions) is based on the Desikan-Killiany atlas (Desikan et al., 2006). >> means "much greater than". 
Table 2. Testing the robustness of the procedure to establish the trade-off between small-world properties and cortical resolution.

\begin{tabular}{|c|c|c|c|c|c|c|c|c|c|c|}
\hline $\begin{array}{c}\text { Range of } \\
\text { cortical }\end{array}$ & OS & & $\mathrm{OS}_{-1}$ & & $\mathrm{OS}_{-2}$ & & $\mathrm{OS}_{-3}$ & & $\mathrm{OS}_{-4}$ & \\
\hline & $\sigma$ & $\mathrm{E}_{\mathrm{lg}}$ & $\sigma$ & $\mathrm{E}_{\mathrm{lg}}$ & $\sigma$ & $\mathrm{E}_{\mathrm{lg}}$ & $\sigma$ & $\mathrm{E}_{\mathrm{lg}}$ & $\sigma$ & $\mathrm{E}_{\mathrm{lg}}$ \\
\hline $66-1494$ & 599 & 571 & 599 & 541 & 599 & 571 & 599 & 571 & 599 & 541 \\
\hline $108-1357$ & 599 & 571 & 599 & 541 & 599 & 541 & 599 & 571 & 599 & 541 \\
\hline $136-1243$ & 599 & 541 & 599 & 541 & 599 & 541 & 376 & 541 & 376 & 541 \\
\hline $157-1031$ & 599 & 541 & 599 & 541 & 599 & 541 & 599 & 541 & 376 & 541 \\
\hline $198-990$ & 599 & 541 & 599 & 541 & 599 & 541 & 599 & 571 & 376 & 541 \\
\hline $229-951$ & 599 & 571 & 599 & 571 & 599 & 571 & 599 & 571 & 599 & 571 \\
\hline $347-879$ & 599 & 571 & 599 & 571 & 599 & 571 & 599 & 571 & 599 & 571 \\
\hline $376-849$ & 599 & 644 & 599 & 644 & 599 & 644 & 599 & 571 & 599 & 571 \\
\hline $496-808$ & 599 & 599 & 599 & 625 & 599 & 571 & 599 & 571 & 599 & 571 \\
\hline
\end{tabular}

OS, optimal sparsity; $\mathrm{OS}_{-\mathrm{n}}, \mathrm{n}^{\text {th }}$ sparsity value closer to the optimal sparsity. 
Tabla 3. Anatomical location of top hubs in four representative cortical scales.

\begin{tabular}{|c|c|c|c|c|c|c|c|}
\hline$\frac{\text { \# Cortical areas }}{66}$ & \multicolumn{7}{|c|}{ Cortical hubs } \\
\hline Atlas location & $\begin{array}{l}\text { L. } \\
\text { Mid. } \\
\text { Front. } \\
\text { BA } 11\end{array}$ & $\begin{array}{c}\text { L. } \\
\text { Parahip. } \\
\text { BA } 36\end{array}$ & $\begin{array}{c}\text { L. } \\
\text { Cingulat. } \\
\text { BA } 24\end{array}$ & $\begin{array}{c}\text { L. } \\
\text { Sup. } \\
\text { Temp. } \\
\text { BA } 22\end{array}$ & & & \\
\hline Degree (Betw.) & $8(2.6)$ & $8(2.3)$ & $8(2.2)$ & $8(2.1)$ & & & \\
\hline \multicolumn{8}{|l|}{108} \\
\hline Atlas location & $\begin{array}{c}\text { R. } \\
\text { Cuneus } \\
\text { BA } 17\end{array}$ & $\begin{array}{l}\text { L. } \\
\text { Mid. } \\
\text { Occip. } \\
\text { BA } 19\end{array}$ & $\begin{array}{c}\text { L. } \\
\text { Mid. } \\
\text { Front. } \\
\text { BA 11,46 }\end{array}$ & $\begin{array}{c}\text { R. } \\
\text { Precent. } \\
\text { BA } 4\end{array}$ & $\begin{array}{c}\text { L. } \\
\text { Cuneus } \\
\text { BA } 17\end{array}$ & & \\
\hline Degree (Betw.) & $13(2.6)$ & $13(2.5)$ & $\begin{array}{c}13(2.2) \\
13(2)\end{array}$ & $13(2.1)$ & $13(1.9)$ & & \\
\hline \multicolumn{8}{|l|}{599 (optimal) } \\
\hline Atlas location & $\begin{array}{c}\text { R. } \\
\text { Inf. } \\
\text { Occipit. } \\
\text { BA } 18\end{array}$ & $\begin{array}{c}\text { L. } \\
\text { Sup. } \\
\text { Front. } \\
\text { BA } 8\end{array}$ & $\begin{array}{c}\text { L. } \\
\text { Inf. } \\
\text { Occipit. } \\
\text { BA } 19\end{array}$ & $\begin{array}{c}\text { L. } \\
\text { Postcent. } \\
\text { BA } 2\end{array}$ & $\begin{array}{l}\text { L. } \\
\text { Inf. } \\
\text { Front. } \\
\text { BA } 47\end{array}$ & $\begin{array}{c}\text { R. } \\
\text { Inf. } \\
\text { Front. } \\
\text { BA } 10\end{array}$ & $\begin{array}{c}\text { L. } \\
\text { Sup. } \\
\text { Temp. } \\
\text { BA } 39\end{array}$ \\
\hline Degree (Betw.) & 67 (1.9) & 67 (1.9) & 67 (1.9) & $65(1.9)$ & $66(1.9)$ & $65(1.8)$ & $61(1.8)$ \\
\hline \multicolumn{8}{|l|}{1494} \\
\hline Atlas location & $\begin{array}{c}\text { R. } \\
\text { Postcent. } \\
\text { BA } 43\end{array}$ & $\begin{array}{l}\text { R. } \\
\text { Mid. } \\
\text { Front. } \\
\text { BA } 10\end{array}$ & $\begin{array}{c}\text { R. } \\
\text { Precun. } \\
\text { BA } 7\end{array}$ & $\begin{array}{c}\text { L. } \\
\text { Inf. } \\
\text { Pariet. } \\
\text { BA } 40\end{array}$ & $\begin{array}{c}\text { L. } \\
\text { Mid. } \\
\text { Front. } \\
\text { BA 8,10 }\end{array}$ & & \\
\hline Degree (Betw.) & 161(1.9) & $\begin{array}{l}153(1.8) \\
155(1.8) \\
153(1.7)\end{array}$ & $157(1.8)$ & $\begin{array}{l}161(1.7) \\
157(1.7) \\
158(1.7)\end{array}$ & $\begin{array}{l}162(1.7) \\
152(1.7)\end{array}$ & & \\
\hline
\end{tabular}

Abbreviations: Betw, Betweeness; L, Left; R, Right; Sup, Superior; Mid, Middle; Inf, Inferior; Front, Frontal; Parahippocamp, Parahippocampal; Cingulat, Cingulated; Temp, Temporal; Occipit, Occipital; Precent, Precentral; Postcent, Postcentral; Paracent, Paracentral; Precun, Precuneus; Pariet, Parietal; BA, Brodmann area. 


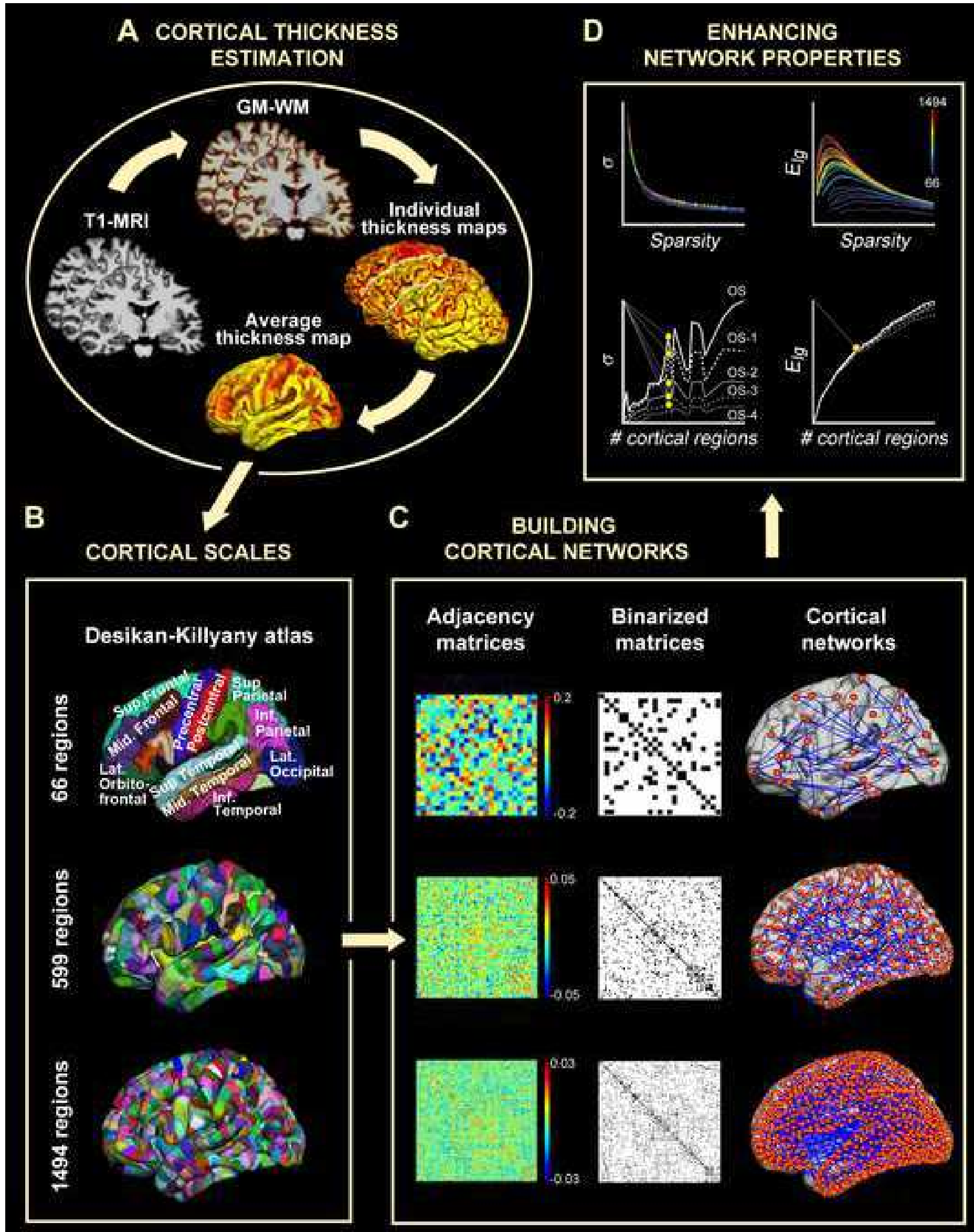


9. Figure2

Click here to download high resolution image

A

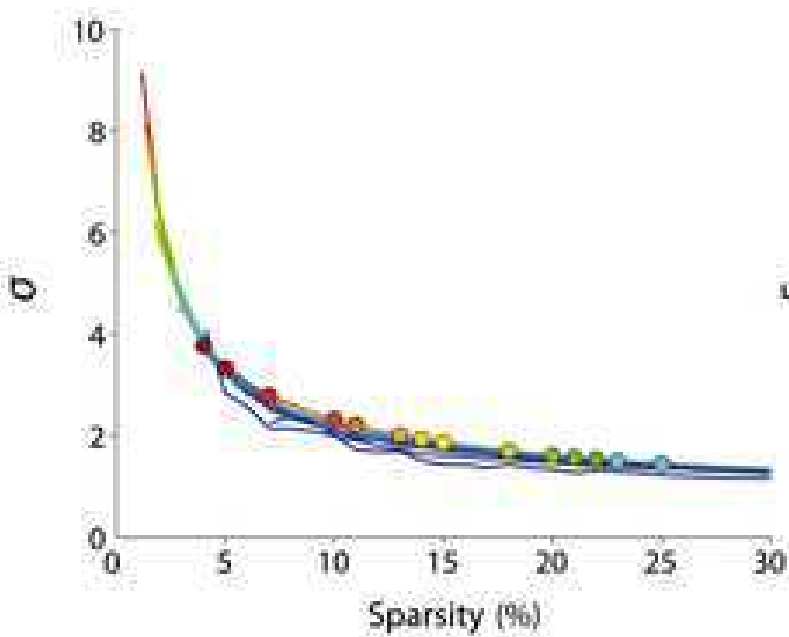

B

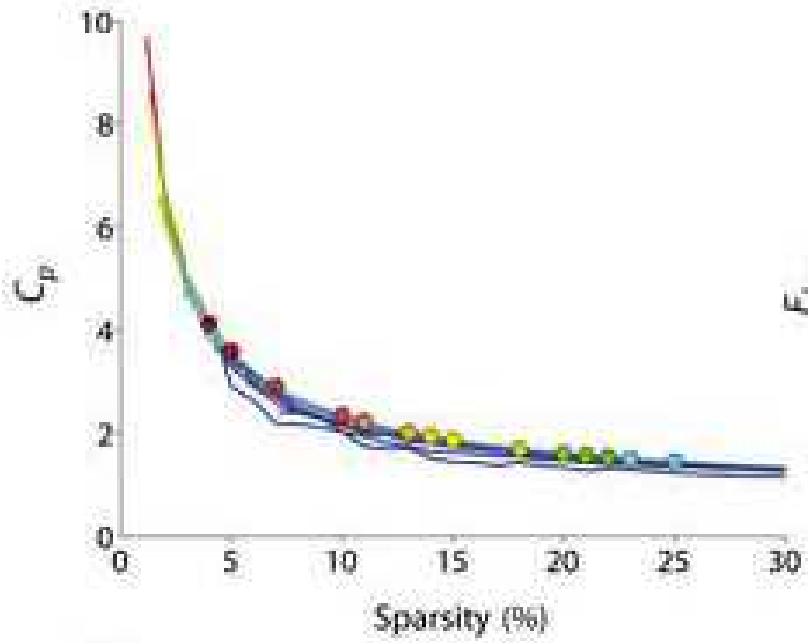

C

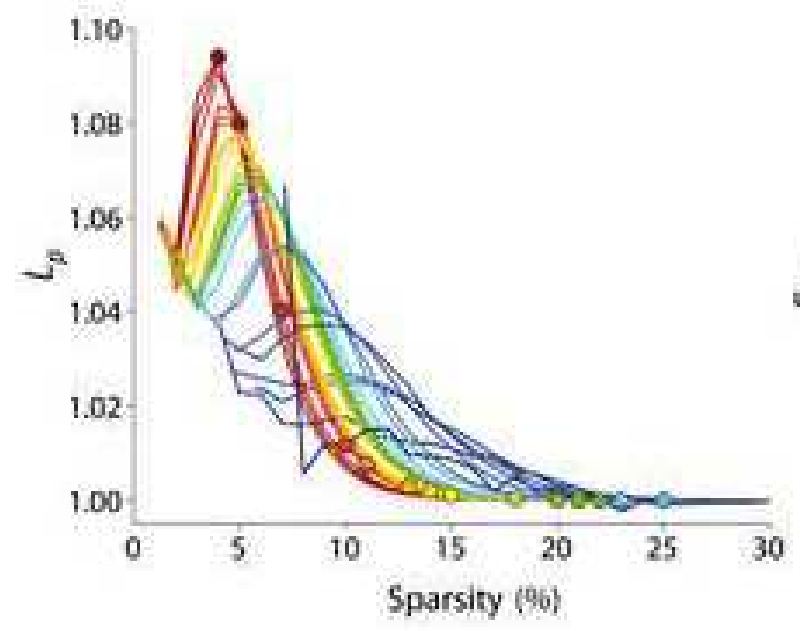

D

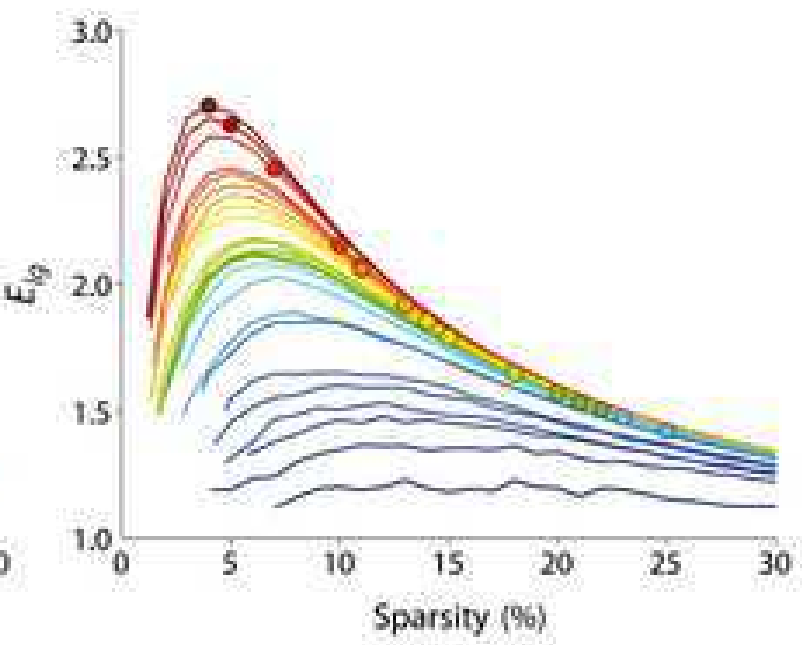

E

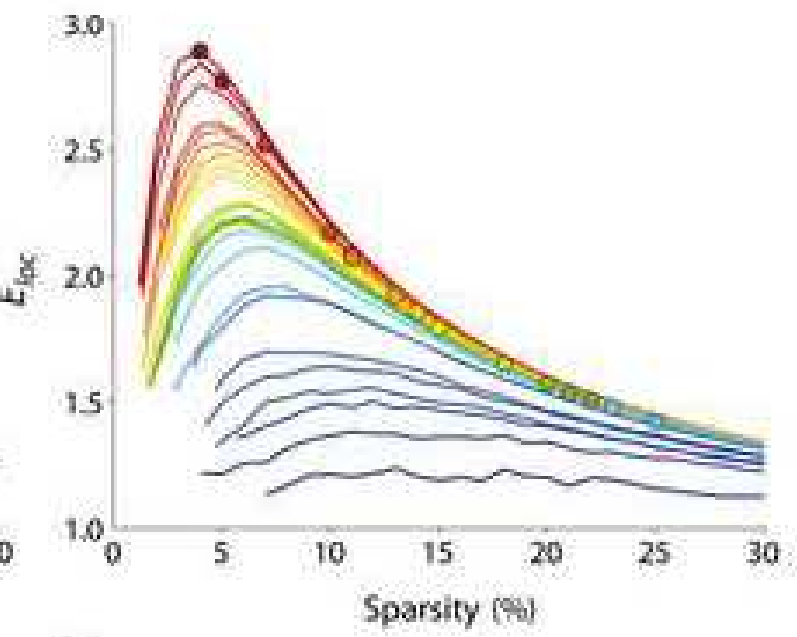

F

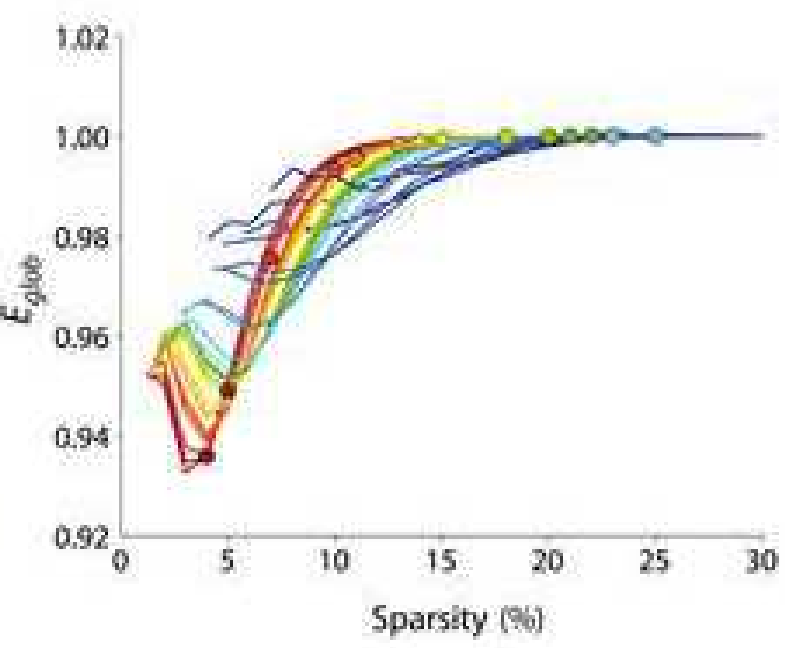

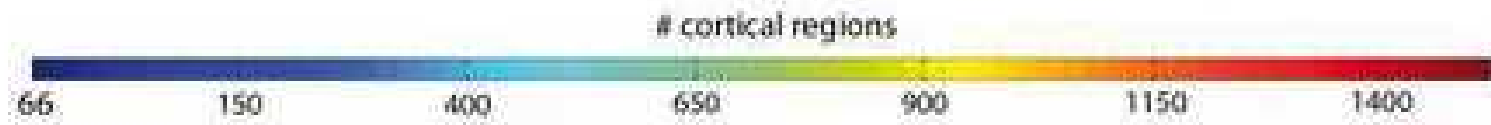




\section{Figure 3}

Click here to download high resolution image
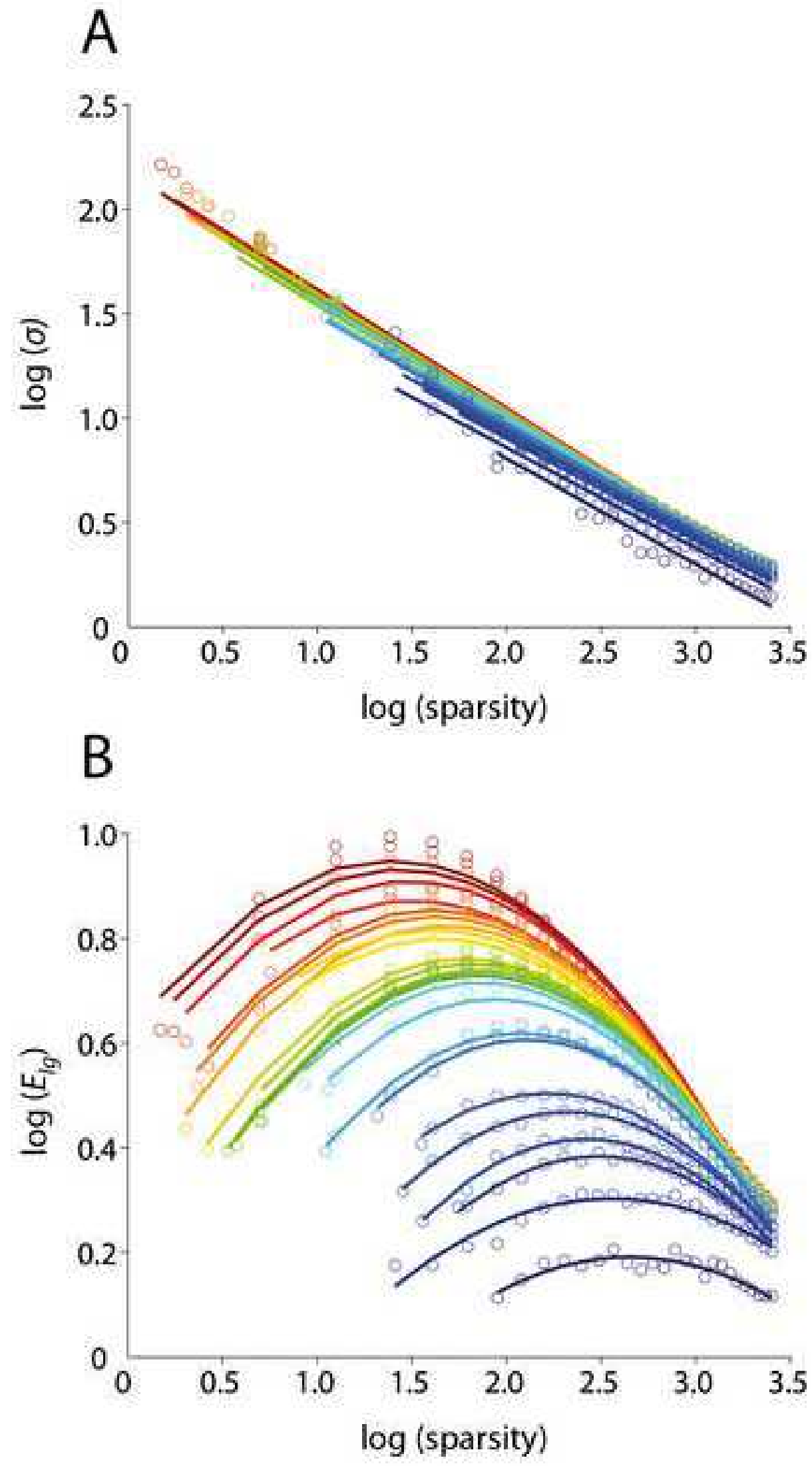

\# cortical regions

\begin{tabular}{lllllll}
\hline 66 & 150 & 400 & 650 & 900 & 1150 & 1400
\end{tabular}



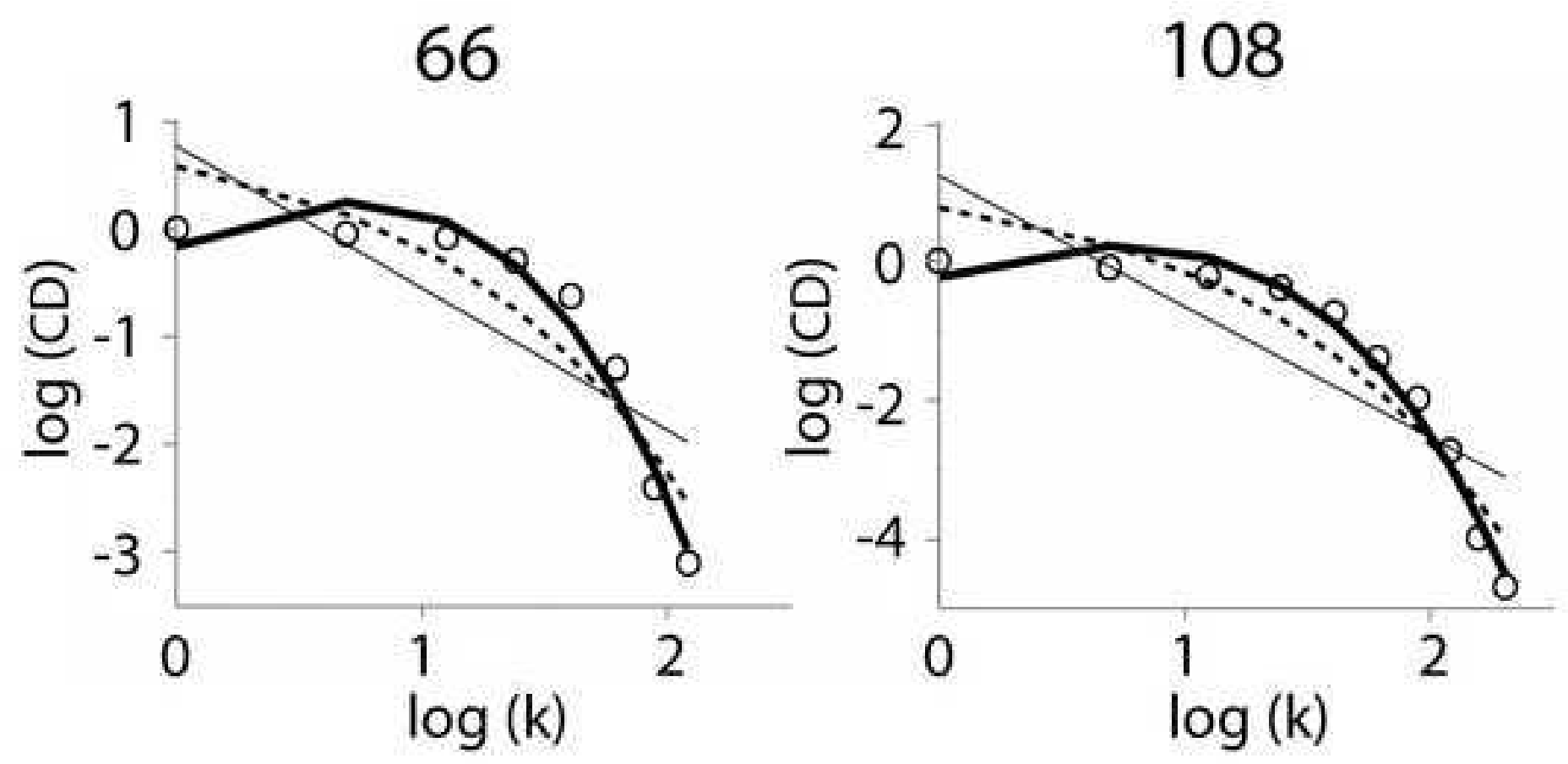

599
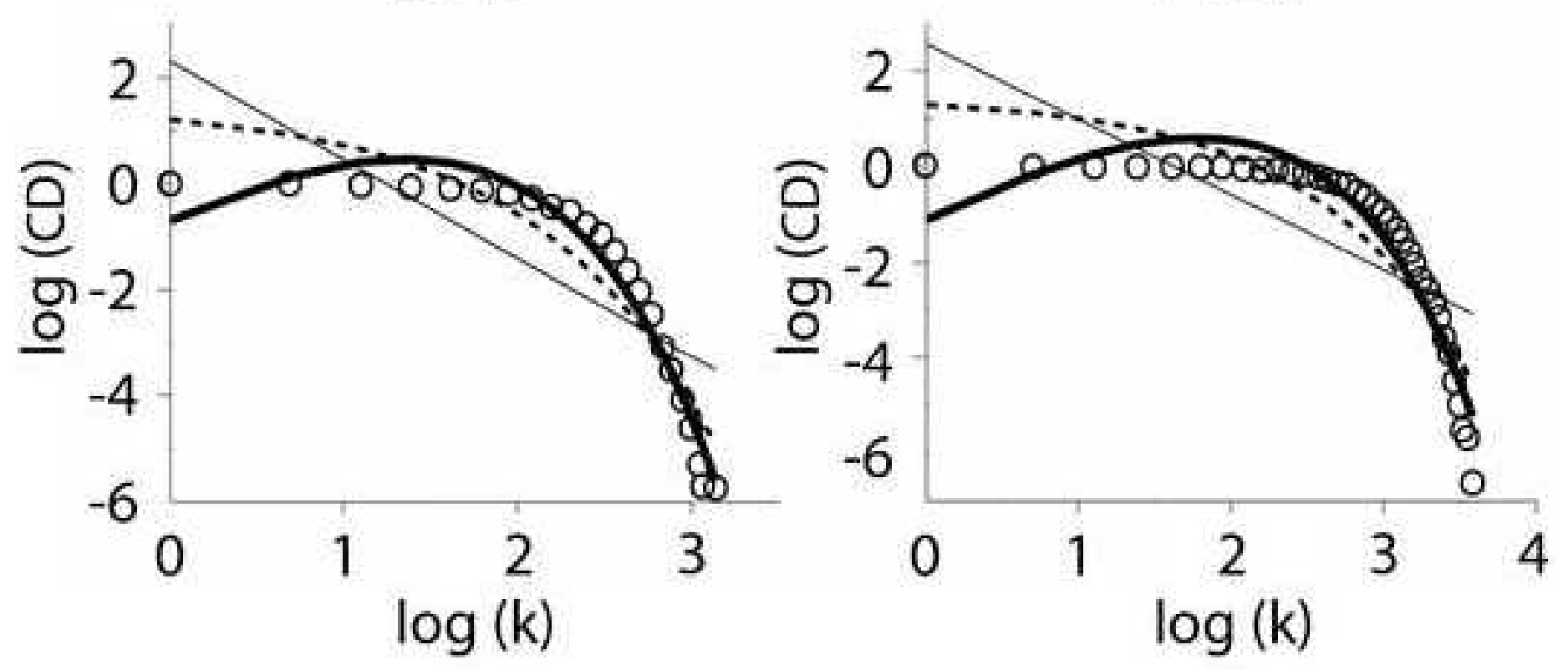

1494

$\log (k)$ 
A
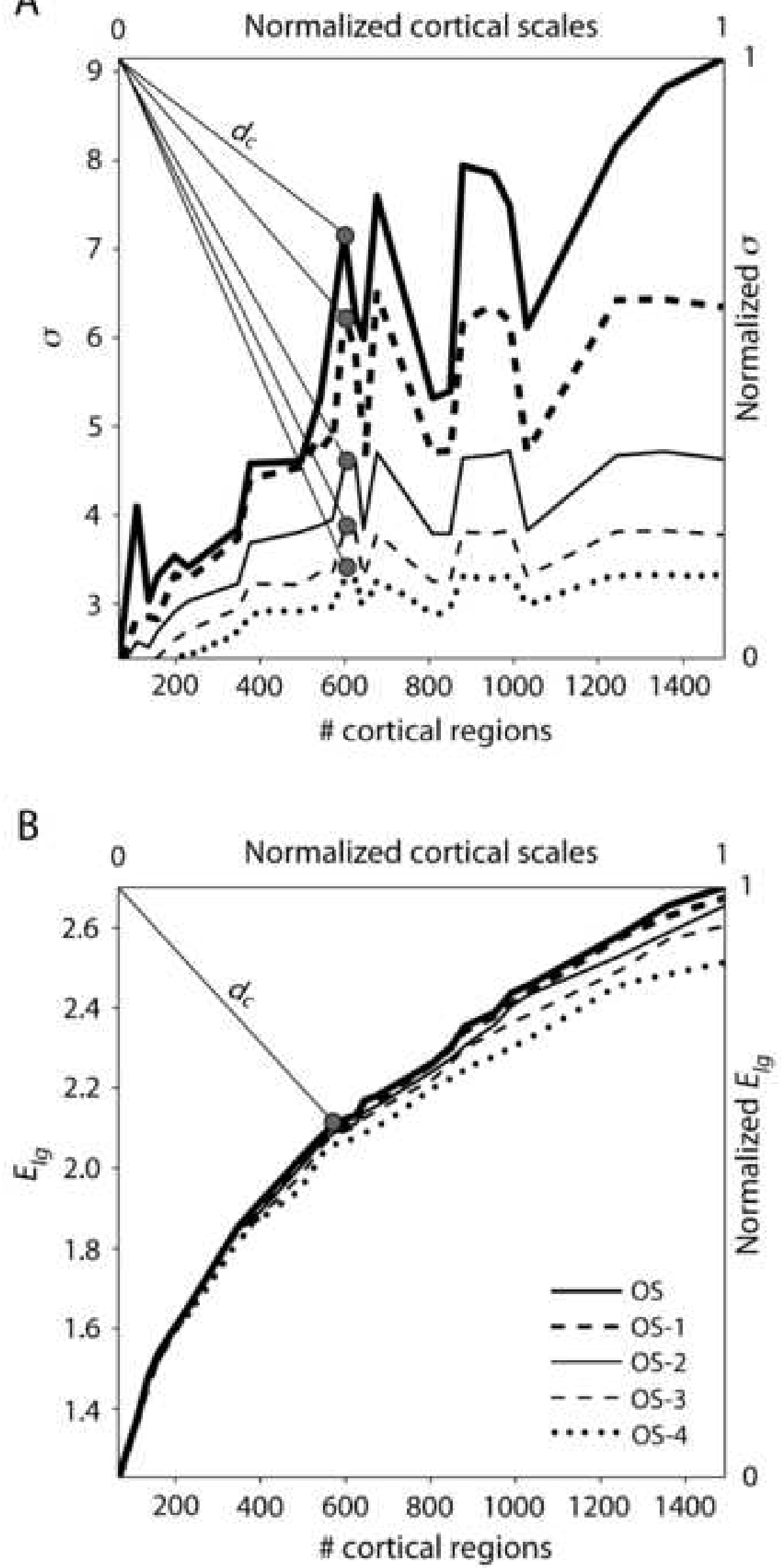
A
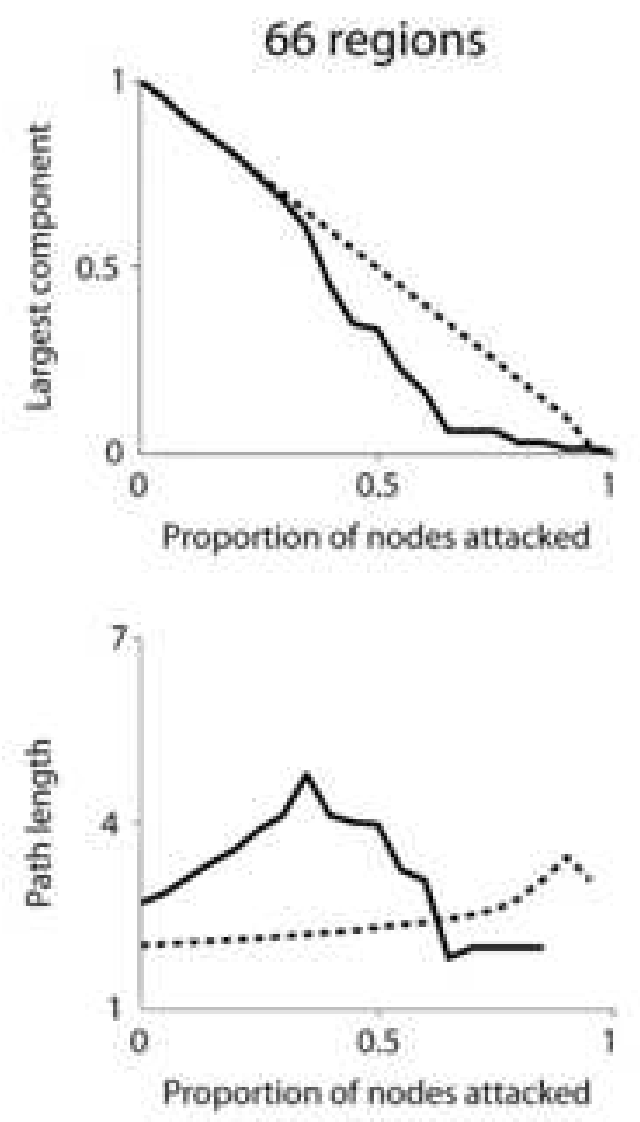

B
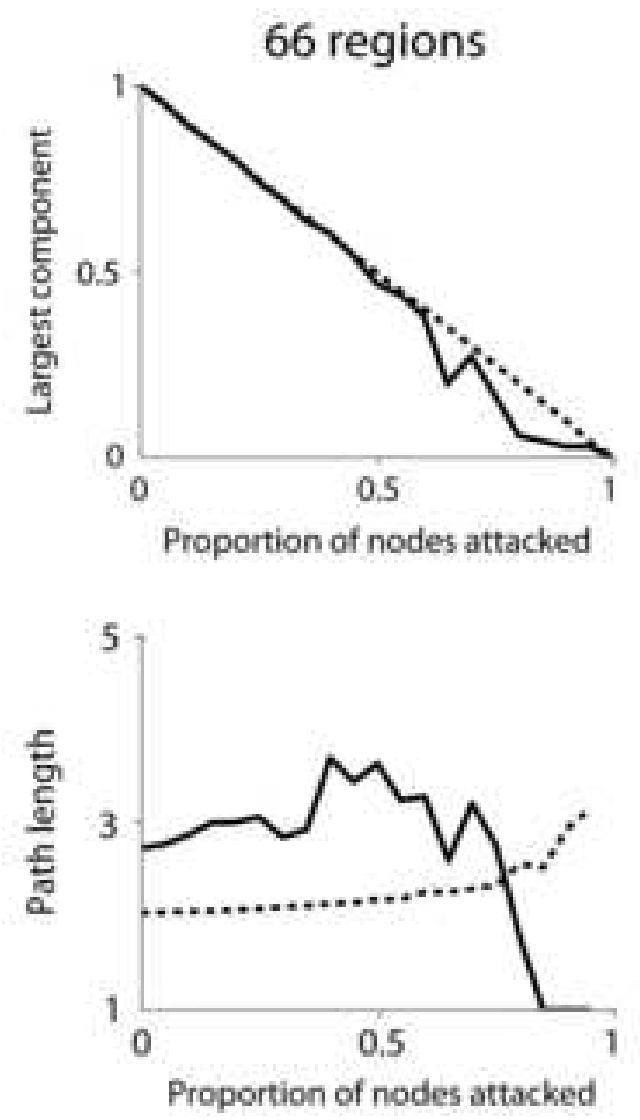

Targeted attack
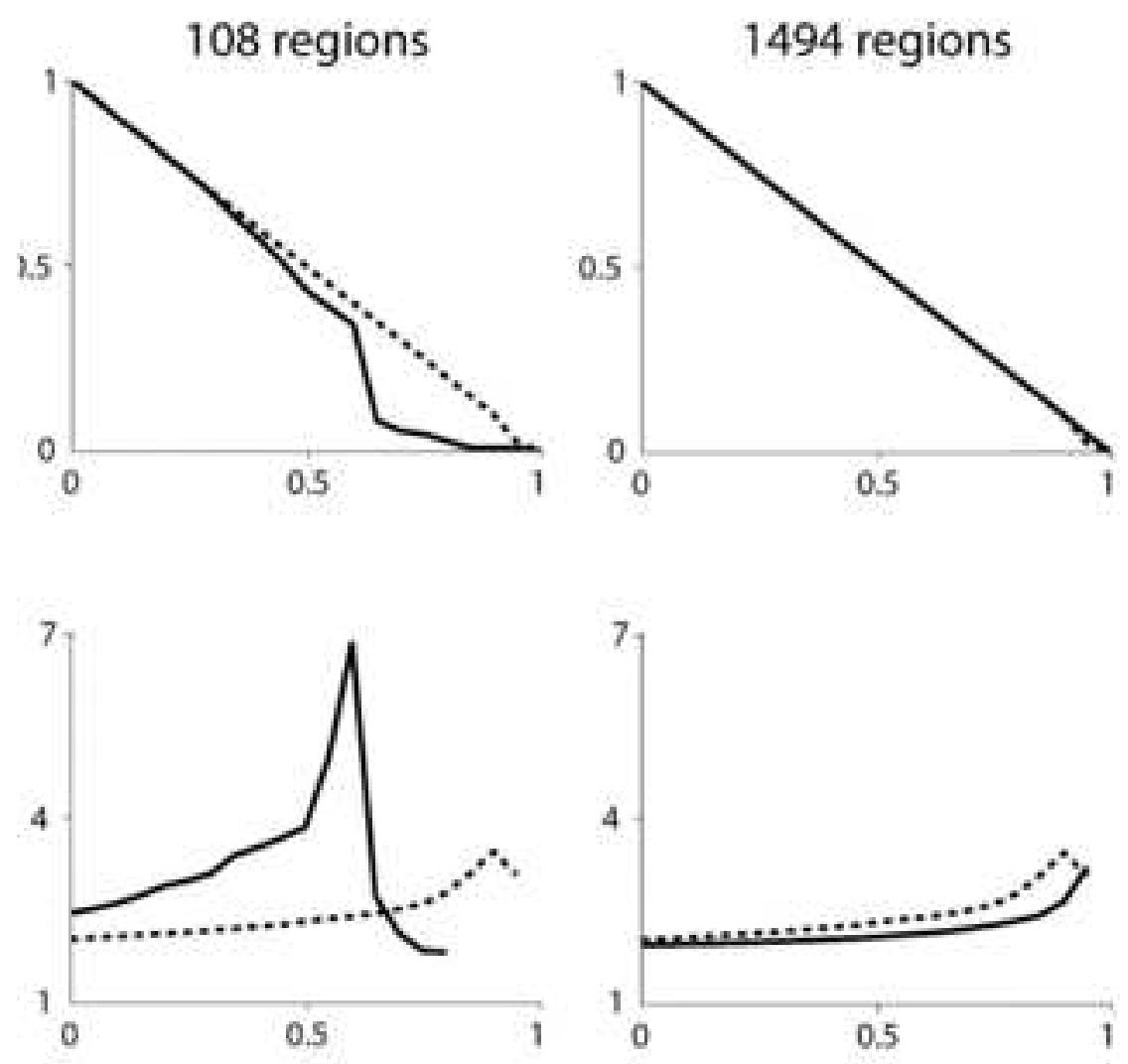

Random attack
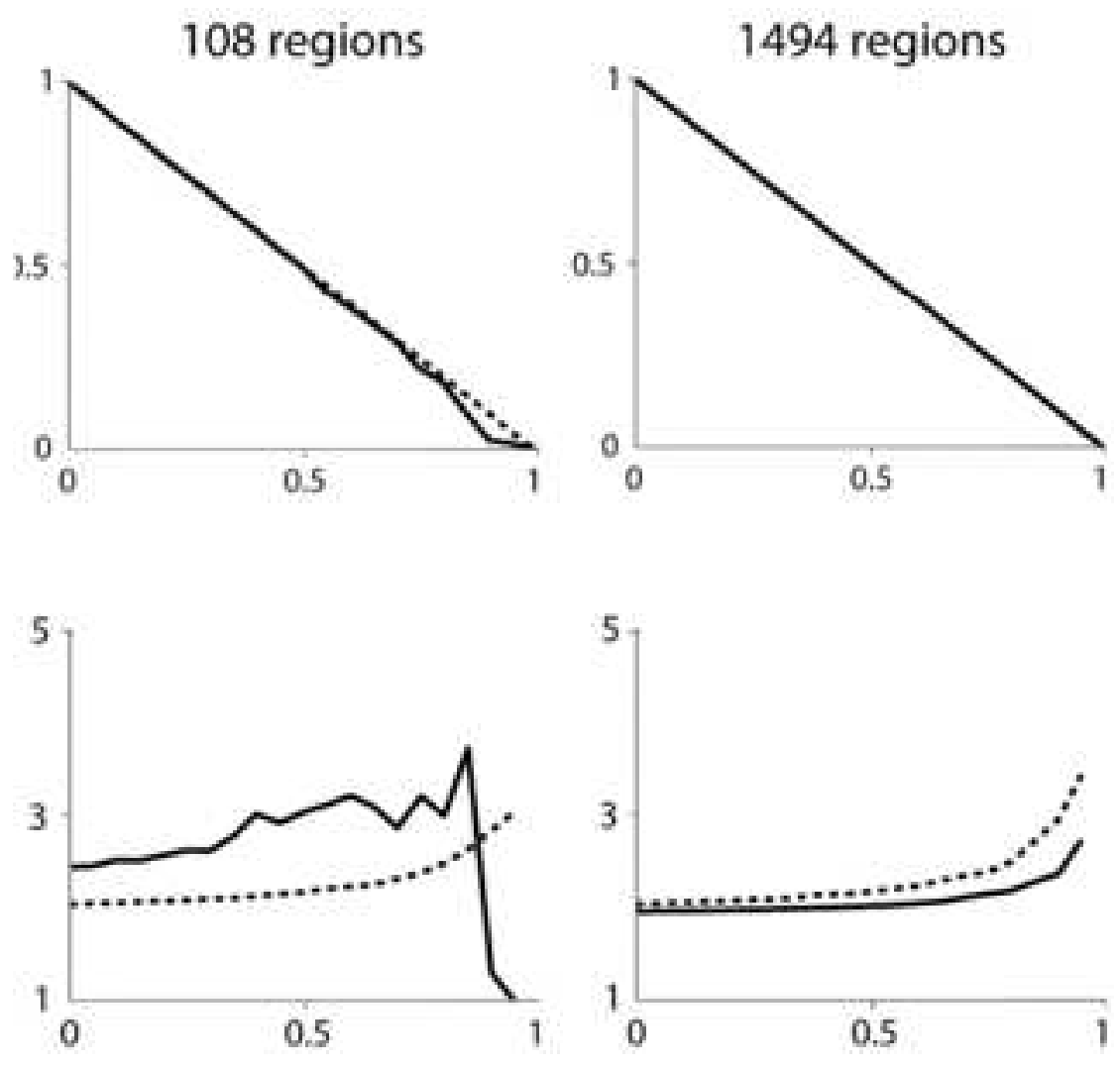
Click here to download 10. Supplementary Material: Supplementary material.doc

10. Supplementary Material
Click here to download 10. S

10. Supplementary Material: Supplementary material.doc 